\title{
Extracting many-body color charge correlators in the proton from exclusive DIS at large Bjorken $x$
}

\author{
Adrian Dumitru* \\ Department of Natural Sciences, Baruch College, CUNY, 17 Lexington Avenue, \\ New York, New York 10010, USA \\ and The Graduate School and University Center, The City University of New York, \\ 365 Fifth Avenue, New York, New York 10016, USA \\ Gerald A. Miller ${ }^{\dagger}$ \\ Department of Physics, University of Washington, Seattle, Washington 98195-1560, USA \\ Raju Venugopalan \\ Physics Department, Brookhaven National Laboratory, Building 510A, Upton, New York 11973, USA
}

(Received 22 August 2018; published 8 November 2018)

\begin{abstract}
We construct a general QCD light front formalism to compute many-body color charge correlators in the proton. These form factors can be extracted from deeply inelastic scattering measurements of exclusive final states in analogy to electromagnetic form factors extracted in elastic electron scattering experiments. Particularly noteworthy is the potential to extract a novel odderon form factor, either indirectly from exclusive $J / \Psi$ measurements or directly from exclusive measurements of the $\eta_{c}$ or tensor mesons at large Bjorken $x$. Besides the intrinsic information conveyed by these color charge correlators on the spatiotemporal tomography at the subfemtoscopic scale at large $x$, the corresponding cumulants extend the domain of validity of McLerran-Venugopalan type weight functionals from small $x$ and large nuclei to nucleons and light nuclei at large $x$, as well as to nonzero momentum transfer. This may significantly reduce nonperturbative systematic uncertainties in the initial conditions for QCD evolution equations at small $x$ and could be of strong relevance for the phenomenology of present and future collider experiments.
\end{abstract}

DOI: 10.1103/PhysRevD.98.094004

\section{INTRODUCTION}

The increasing availability of high energies and high luminosities at fixed target and collider experiments [1,2] allows for unprecedented access to the internal transverse spatial and momentum distributions of color charge distributions inside nucleons and in nuclei. The standard framework [3] is that of Wigner distributions [4] that allow simultaneous knowledge of both spatial and momentum aspects of the nucleon wave function. Knowledge of the Wigner distributions allows the construction of generalized parton distributions (GPDs) [5-11] and transverse momentum distributions (TMDs) [12-17] that are generalizations of the usual collinear parton distributions. The GPDs

\footnotetext{
*adrian.dumitru@baruch.cuny.edu †miller@uw.edu

raju@bnl.gov
}

Published by the American Physical Society under the terms of the Creative Commons Attribution 4.0 International license. Further distribution of this work must maintain attribution to the author(s) and the published article's title, journal citation, and DOI. Funded by SCOAP. provide information on the spatial tomography of the nucleon and TMDs allow for its momentum tomography.

These various distributions are very valuable. Our aim here is to introduce a complementary approach employing the Hamiltonian light front formalism in light-cone gauge that allows essential insight into the dynamics of color charges in nucleons and nuclei. In this framework, color charge densities, and higher cumulants of these, can be defined and expressed as matrix elements of nonperturbative boost-invariant light front Fock-space wave functions of the QCD Hamiltonian. The corresponding form factors can be related to physical observables; these are the exclusive final states measured in deeply inelastic scattering (DIS) experiments. The information on color charge distributions extracted from such exclusive DIS measurements will be closely analogous to the information gathered on electric charge and magnetization distributions from form factors measured in elastic scattering of electrons by nucleons and nuclei [18-21].

However because the QCD coupling $\alpha_{S}$ is much stronger than the QED fine structure constant, exclusive DIS experiments provide more information on color charge 
distributions, and higher cumulants of these, than elastic scattering experiments. Though it is true that GPDs and TMDs can be expressed in terms of light front wave functions [22-24], our treatment in terms of color charge densities is novel.

The suite of feasible exclusive DIS final states is a rich source of information on many-body parton correlations with variations in $x_{\mathrm{Bj}}$ and $Q^{2}$ and can be expected to lead to an understanding of the internal spatial color charge structure of nucleons. The possible modification of this structure in nuclei could be important for understanding the EMC effect in DIS and nucleon-nucleon short range correlations in nuclei $[25,26]$. Also very intriguing is the possibility of comparing the color charge form factors to be discussed here with those that are now beginning to be extracted from lattice QCD computations [27].

An attractive feature of the Hamiltonian light front framework is that the color charge form factors extracted in DIS can be employed to compute cross sections in hadron-hadron and hadron-nucleus scattering. The usefulness of such color charge form factors is known for QCD in the Regge limit of high energy scattering, with momentum resolution scales $Q^{2} \gg \Lambda_{\mathrm{QCD}}^{2}$ and $x_{\mathrm{Bj}} \sim Q^{2} / s \rightarrow 0$, with $s$ representing the squared center of mass energy in the experiment, as understood in the color glass condensate (CGC) [28-31]. This is an effective field theory of the Regge limit of QCD that is formulated on the light front, with all the nontrivial information regarding multigluon correlations contained in a gauge invariant weight functional $W[\rho]$ that plays the role of a density matrix. Here, $\rho$ represents the color charge density of large $x$ partons coupled to small $x$ gluon fields.

This weight functional was first derived by McLerran and Venugopalan (MV) [32-34], who also outlined the elements of the CGC effective field theory using light front arguments. They argued that for a large nucleus $A$, a probe of transverse size $\sim 1 / Q$ couples coherently (for $x \ll A^{-1 / 3}$ ) along its path length to partons confined to nucleons in the nucleus. While on average, the probe sees no net color charge, the physics of random walks indicates that it will see large fluctuations of the color charge and therefore, by the central limit theorem, $W[\rho]$ will be Gaussian. These statements can be formulated with mathematical rigor $[35,36]$.

The variance of the Gaussian is the color charge squared per unit area $\mu_{\mathrm{MV}}^{2} \propto A^{1 / 3}$. In the large $A$ limit $\mu_{\mathrm{MV}}^{2} \gg \Lambda_{\mathrm{QCD}}^{2}$, so that the CGC is a weakly coupled EFT that allows for systematic computation of multigluon correlation functions that capture the physics underlying the phenomenon of gluon saturation [37,38] in the high energy limit. The building block of gluon radiation, the WeizsäckerWilliams distribution, is screened at the scale $Q_{S}^{2} \propto \mu_{\mathrm{MV}}^{2}$ $[35,39,40]$, and one recovers the phenomenologically successful Glauber-Mueller dipole model $[41,42]$ of gluon saturation [43-45].
The MV model does not describe the small $x$ evolution of the color source densities that arise from the $\alpha_{S} \ln (x) \sim$ $O(1)$ enhanced bremsstrahlung of gluons. This is given by the Jalilian-Marian, Iancu, McLerran, Weigert, Leonidov, Kovner (JIMWLK) equation that describes the functional renormalization group evolution of $W[\rho]$ with decreasing $x$ [46-49]. This functional equation gives the BalitskyJIMWLK hierarchy [50,51]. The equivalent functional Langevin equation was solved numerically $[52,53]$. In the limit of large $N_{c}$, and large $A$, the lowest equation in this hierarchy, describing the $x$ evolution of "dipole" twopoint correlators of lightlike Wilson lines, has a closed form expression, the Balitsky-Kovchegov (BK) equation $[44,50]$, which reduces to the Balitsky, Fadeev, Kuraev, Lipatov (BFKL) equation [54,55] if the density of sources is sufficiently low.

Remarkably, as first conjectured in [56], numerical simulations of the functional Langevin equation demonstrate that the hierarchy of correlators is to good approximation solved by a Gaussian $W[\rho]$ [57], with $\mu_{\mathrm{MV}}^{2} \rightarrow$ $\mu_{\mathrm{JIMWLK}}^{2}\left(x, k_{\perp}\right)$, where $\mu_{\mathrm{JIMWLK}}^{2}\left(x, k_{\perp}\right)$ is given by the solution of the BK equation. This Gaussian effective theory provides a quantitative phenomenology of electron-proton collisions at HERA [58-60]. Further, the formulation of the CGC EFT in the language of color source densities allows a first-principles formulation of multiparticle production in QCD at small $x$ [61-67].

The initial conditions for BK/JIMWLK evolution are given by the MV model which, as noted, is formulated for large nuclei. Here we are concerned with the nucleon at large $x$. In this case, the central limit theorem is not applicable and the color charge form factors of the proton can reasonably be expected to be very different than in the MV model. Therefore a first-principles computation of these form factors is in order. Such a computation is of intrinsic interest and can help constrain the systematic uncertainties in the QCD evolution of color charge distributions in the proton arising from the initial conditions. The spatial distributions of color charge density in the proton are also of great topical interest because of the unexpected long range azimuthally collimated "ridge" multiparticle correlations measured at RHIC and LHC [68]; the latter may depend sensitively on the former [69-75]. Several models have been constructed to incorporate spatial nucleon color charge distributions in describing these data. However, they are constrained in varying degrees by systematic uncertainties in the initial conditions [76-78].

Here we develop a light front Hamiltonian framework that can be used to compute color charge form factors in nucleons and nuclei. The light front formalism we will employ is standard; see for instance [79]. We focus on the simple problem of constructing quadratic and cubic combinants of a three quark Fock state at large $x$. The color charge combinants can alternatively be expressed in terms of color charge form factors. We will discuss how information on these form factors can be cleanly extracted in exclusive 
DIS measurements of vector and tensor mesons at large $x$. An interesting possibility is the extraction of a novel odderon color charge form factor in such measurements [80]. As we will discuss, large $x$ DIS exclusive measurements should be particularly sensitive to the odderon. This is of topical interest in light of recent claims that the TOTEM experiment may have found evidence of odderon exchange in protonproton elastic scattering at the highest LHC energies [81].

This paper is organized as follows. In Sec. II, we begin by displaying the light front wave function for the proton, focusing immediately on the three valence quark component of the wave function. The extension to higher Fock states would be straightforward, but more involved. We also establish the notations and conventions to be employed in the rest of the paper. We then develop in Sec. III, in successive subsections, the general framework to compute light front color charge densities for the valence states, and the computation of the expectation values of quadratic and cubic color charge operators. In the last of these subsections, we compare our results to the MV model and demonstrate the relation between the gluon distribution in the proton and a quadratic correlator of color charge densities. The relation of the corresponding color charge form factors to exclusive heavy quark pair production in DIS is discussed in Sec. IV. In particular, we show that $J / \Psi$ production is sensitive to both a quadratic "Pomeron" color charge form factor and the cubic odderon color charge form factor. In contrast, $\eta_{c}$ or tensor meson production depends only on the odderon form factor. In the concluding section, we will further discuss the prospects of odderon discovery in DIS experiments in light of prior searches. We will also discuss more generally the prospects for quantitative constraints on the quadratic and cubic color charge form factors from DIS data at large $x_{\mathrm{Bj}}$. We shall also outline the next steps both on further theoretical development of this framework and in quantitative comparison and predictions for measurements at extant and future experiments. The paper contains two Appendices. In Appendix A, we discuss the color charge density operator in the limit of large longitudinal momenta. In Appendix B, we provide some details of the computation of the odderon form factor.

\section{THE LIGHT FRONT PROTON WAVE FUNCTION: NOTATION AND CONVENTIONS}

In this section, we shall introduce our notation and conventions for the proton wave function on the light front. These closely follow Refs. [82,83]. The light front wave function of an unpolarized on-shell proton with fourmomentum $P^{\mu}=\left(P^{+}, P^{-}, \vec{P}_{\perp}\right)$ can be expressed as

$$
|P\rangle=\int \mathrm{dPS}_{n} \sum_{n} \psi_{n}|n\rangle,
$$

where $|n\rangle$ are the Fock space basis vectors of the light front Hamiltonian, $\psi_{n}=\langle n \mid P\rangle$ is the amplitude for a particular Fock state $|n\rangle$ in the proton and dPS denotes the $n$-body phase space for $|n\rangle$. If the proton light front wave function is dominated by its valence quark state, as is the case at large values of Bjorken $x$, it is given explicitly as

$$
\begin{aligned}
|P\rangle= & \frac{1}{\sqrt{6}} \int \frac{\mathrm{d} x_{1} \mathrm{~d} x_{2} \mathrm{~d} x_{3}}{\sqrt{x_{1} x_{2} x_{3}}} \delta\left(1-x_{1}-x_{2}-x_{3}\right) \int \frac{\mathrm{d}^{2} k_{1} \mathrm{~d}^{2} k_{2} \mathrm{~d}^{2} k_{3}}{\left(16 \pi^{3}\right)^{3}} 16 \pi^{3} \delta\left(\vec{k}_{1}+\vec{k}_{2}+\vec{k}_{3}\right) \\
& \times \sum_{\lambda_{1}, \lambda_{2}, \lambda_{3}} \psi_{3}\left(p_{1}, \lambda_{1}, p_{2}, \lambda_{2}, p_{3}, \lambda_{3}\right) \sum_{i_{1}, i_{2}, i_{3}} \epsilon_{i_{1} i_{2} i_{3}}\left|p_{1}, i_{1}, \lambda_{1} ; p_{2}, i_{2}, \lambda_{2} ; p_{3}, i_{3}, \lambda_{3}\right\rangle .
\end{aligned}
$$

The three on-shell quark momenta are specified by their light-cone momenta $p_{i}^{+}=x_{i} P^{+}$and their transverse momenta $^{1} \vec{p}_{i}=x_{i} \vec{P}_{\perp}+\vec{k}_{i}$. Hence the $\vec{k}_{i}$ can be interpreted as the transverse momenta of the valence quarks relative to the proton. In addition to color, denoted by $i_{i}$, the quark Fock state also carries flavor and helicity quantum numbers which are collectively denoted as $\lambda_{i}$. The valence Fock state wave function in color space belongs to the product space obtained from the direct product of three triplet color spaces: $\left|i_{1} ; i_{2} ; i_{3}\right\rangle=\left|i_{1}\right\rangle \otimes\left|i_{2}\right\rangle \otimes\left|i_{3}\right\rangle$. The Levi-Cività tensor in Eq. (2) projects the product of three fundamental representations onto the totally antisymmetric $\mathrm{SU}(3)$ singlet; a $\mathrm{SU}(3)$ transformation $U$ of $\left.\epsilon_{i_{1} i_{2} i_{3}}\left|i_{1}\right\rangle\left|i_{2}\right\rangle i_{3}\right\rangle$ gives

\footnotetext{
${ }^{1}$ For a lighter notation we often suppress the $\perp$ subscript on quark transverse momenta.
}

$\epsilon_{i_{1} i_{2} i_{3}} U_{j_{1} i_{1}} U_{j_{2} i_{2}} U_{j_{3} i_{3}}\left|j_{1}\right\rangle\left|j_{2}\right\rangle\left|j_{3}\right\rangle=\epsilon_{j_{1} j_{2} j_{3}}(\operatorname{det} U)\left|j_{1}\right\rangle\left|j_{2}\right\rangle\left|j_{3}\right\rangle$,

where det $U=1$ for $U \in \mathrm{SU}(3)$.

The amplitude $\psi_{3}$ in Eq. (2) is symmetric under exchange of any two of its arguments and is normalized according to

$$
\begin{aligned}
& \int \mathrm{d} x_{1} \mathrm{~d} x_{2} \mathrm{~d} x_{3} \delta\left(1-x_{1}-x_{2}-x_{3}\right) \\
& \times \int \frac{\mathrm{d}^{2} k_{1} \mathrm{~d}^{2} k_{2} \mathrm{~d}^{2} k_{3}}{\left(16 \pi^{3}\right)^{3}}\left(16 \pi^{3}\right) \delta\left(\vec{k}_{1}+\vec{k}_{2}+\vec{k}_{3}\right) \sum_{\lambda_{1}, \lambda_{2}, \lambda_{3}}\left|\psi_{3}\right|^{2}=1 .
\end{aligned}
$$

Note that $\psi_{3}$ vanishes when the set $\left\{\lambda_{1}, \lambda_{2}, \lambda_{3}\right\}$ does not match the corresponding quantum numbers of the proton. 
The normalization of $\psi_{3}$ corresponds to the proton wave function normalization,

$$
\begin{aligned}
\langle K \mid P\rangle & =16 \pi^{3} P^{+} \delta\left(P^{+}-K^{+}\right) \delta\left(\vec{P}_{\perp}-\vec{K}_{\perp}\right) \\
& =16 \pi^{3} \delta(\Delta x) \delta\left(\vec{P}_{\perp}-\vec{K}_{\perp}\right) .
\end{aligned}
$$

For simplicity, throughout the manuscript we take the fractional plus momentum transfer $\Delta x=\left(K^{+}-P^{+}\right) / P^{+}$ to be very small or zero.

The one-particle quark states introduced above are created by the action of the quark creation operator $b_{p, i, \lambda}^{\dagger}$ on the one-particle vacuum $|0\rangle$ :

$$
|p, i, \lambda\rangle=b_{p, i, \lambda}^{\dagger}|0\rangle .
$$

Its Hermitian conjugate transforms an occupied one-particle state to the light front vacuum state,

$$
\begin{gathered}
b_{k, j, \sigma}|p, i, \lambda\rangle=\delta^{j i} \delta^{\sigma \lambda} k^{+} \delta\left(k^{+}-p^{+}\right) 16 \pi^{3} \delta(\vec{k}-\vec{p})|0\rangle \\
\equiv \delta_{k, p}^{j i, \sigma \lambda}|0\rangle, \\
b_{k, j, \sigma}|0\rangle=0 .
\end{gathered}
$$

In Eq. (8), we introduced a shorthand notation $\delta_{k, p}^{j i, \sigma \lambda}$, which we will frequently use throughout the rest of the paper. We shall further also use the shorthand notation,

$$
\begin{gathered}
\delta_{k, p}^{i j} \equiv \delta^{j i} \delta_{k, p} \\
\delta_{k, p} \equiv k^{+} \delta\left(k^{+}-p^{+}\right) 16 \pi^{3} \delta(\vec{k}-\vec{p}) .
\end{gathered}
$$

The quark creation and destruction operators satisfy the anticommutation relation,

$$
\left\{b_{k, j, \sigma}, b_{p, i, \lambda}^{\dagger}\right\}=\delta_{k, p}^{j i, \sigma \lambda}
$$

These relations, along with the convention that $\langle 0 \mid 0\rangle=1$, determine the normalization of one-particle states as

$$
\begin{aligned}
\langle k, j, \sigma \mid p, i, \lambda\rangle & =\left\langle 0\left|b_{k, j, \sigma} b_{p, i, \lambda}^{\dagger}\right| 0\right\rangle \\
& =\left\langle 0\left|\left\{b_{k, j, \sigma}, b_{p, i, \lambda}^{\dagger}\right\}\right| 0\right\rangle=\delta_{k, p}^{j i, \sigma \lambda} .
\end{aligned}
$$

Furthermore,

$$
\left\langle k, j, \sigma\left|b_{q, m, \sigma^{\prime}}^{\dagger} b_{r, n, \lambda^{\prime}}\right| p, i, \lambda\right\rangle=\delta_{k, q}^{j m, \sigma \sigma^{\prime}} \delta_{r, p}^{n i, \lambda \lambda^{\prime}},
$$

and

$$
\left\langle k, j, \sigma\left|b_{q, m, \sigma^{\prime}} b_{r, n, \lambda^{\prime}}^{\dagger}\right| p, i, \lambda\right\rangle=\delta_{q, r}^{m n, \sigma^{\prime} \lambda^{\prime}} \delta_{k, p}^{j i, \sigma \lambda}-\delta_{k, r}^{j n, \sigma \lambda^{\prime}} \delta_{q, p}^{m i, \sigma^{\prime} \lambda} .
$$

With these relations in hand, one can derive matrix elements of density operators and powers thereof.

Before we discuss color charge densities, let us first consider the following operator:

$$
\left[\tilde{\rho}_{q}^{m n}\right]_{1}=\bigotimes_{\ell, \lambda} b_{\ell-q, m, \lambda}^{\dagger} b_{\ell, n, \lambda} .
$$

We have written the integration measure here compactly as

$$
\sum_{\ell, \lambda} \equiv \int_{0}^{\infty} \frac{\mathrm{d} \ell^{+}}{\ell^{+}} \int \frac{\mathrm{d}^{2} \ell}{16 \pi^{3}} \sum_{\lambda}, \quad \oint_{\ell, \lambda} \delta_{q, l}^{i j, \sigma \lambda}=\delta^{i j} .
$$

Setting $\vec{P}_{\perp}=0$ in the incoming proton for simplicity, and employing Eqs. (14) and (12), we obtain the expectation value of the operator defined in Eq. (16) as

$$
\begin{aligned}
\left\langle K\left|\left[\tilde{\rho}_{q}^{m n}\right]_{1}\right| P\right\rangle= & \frac{1}{16 \pi^{3}} \delta^{m n} \int \frac{\mathrm{d} x_{1} \mathrm{~d} x_{2} \mathrm{~d} x_{3}}{\sqrt{x_{1} x_{2} x_{3}}} \delta\left(1-x_{1}-x_{2}-x_{3}\right) \int \mathrm{d}^{2} p_{1} \mathrm{~d}^{2} p_{2} \mathrm{~d}^{2} p_{3} \delta\left(\vec{p}_{1}+\vec{p}_{2}+\vec{p}_{3}\right) \int \frac{\mathrm{d} y_{1} \mathrm{~d} y_{2} \mathrm{~d} y_{3}}{\sqrt{y_{1} y_{2} y_{3}}} \delta\left(1-y_{1}-y_{2}-y_{3}\right) \\
& \times \int \mathrm{d}^{2} k_{1} \mathrm{~d}^{2} k_{2} \mathrm{~d}^{2} k_{3} \delta\left(\vec{k}_{1}+\vec{k}_{2}+\vec{k}_{3}\right) \sum_{\lambda_{1}, \lambda_{2}, \lambda_{3}} \psi_{3}^{*}\left(k_{1}, k_{2}, k_{3}\right) \psi_{3}\left(p_{1}, p_{2}, p_{3}\right) \delta_{k_{1}, p_{1}-q} \delta_{k_{2}, p_{2}} \delta_{k_{3}, p_{3}} .
\end{aligned}
$$

It is implied that $y_{i}, \vec{k}_{i}$ are the momentum fractions and transverse momenta, respectively, of the quarks in the outgoing proton. However, there is a subtlety: the plus momenta of the quarks in the outgoing proton correspond to $k_{i}^{+}=y_{i} K^{+}=$ $y_{i}(1+\Delta x) P^{+}$rather than to $k_{i}^{+}=y_{i} P^{+}$. Therefore, in the arguments of the delta functions originating from the Fock space matrix elements (the last three in the expression above) we have to shift $y_{i} \rightarrow y_{i}(1+\Delta x)$; we also have to shift $\vec{k}_{i} \rightarrow$ $\vec{k}_{i}+y_{i} \vec{K}_{\perp}$ since there is a nonzero transfer of transverse momentum. To simplify the final expression we shall take $\Delta x \rightarrow 0$ so that

$$
\begin{aligned}
\left\langle K\left|\left[\tilde{\rho}_{q}^{m n}\right]_{1}\right| P\right\rangle= & 16 \pi^{3} \delta\left(\vec{q}+\vec{K}_{\perp}\right) \delta\left(x_{q}+\Delta x\right) \delta^{m n} \int \mathrm{d} x_{1} \mathrm{~d} x_{2} \mathrm{~d} x_{3} \delta\left(1-x_{1}-x_{2}-x_{3}\right) \int \frac{\mathrm{d}^{2} p_{1} \mathrm{~d}^{2} p_{2} \mathrm{~d}^{2} p_{3}}{\left(16 \pi^{3}\right)^{2}} \delta\left(\vec{p}_{1}+\vec{p}_{2}+\vec{p}_{3}\right) \\
& \times \sum_{\lambda_{1}, \lambda_{2}, \lambda_{3}} \psi_{3}^{*}\left(k_{1}, k_{2}, k_{3}\right) \psi_{3}\left(p_{1}, p_{2}, p_{3}\right) .
\end{aligned}
$$


In the limit $\Delta x \rightarrow 0$ the arguments of $\psi_{3}^{*}$ are $k_{i}^{+} \simeq x_{i} P^{+}$, $\vec{k}_{1} \simeq \vec{p}_{1}+\left(1-x_{1}\right) \vec{K}_{\perp}, \vec{k}_{2} \simeq \vec{p}_{2}-x_{2} \vec{K}_{\perp}, \vec{k}_{3} \simeq \vec{p}_{3}-x_{3} \vec{K}_{\perp}$. (Note that the flavor and helicity of each quark remains unchanged.)

The prefactor, $16 \pi^{3} \delta\left(\vec{q}+\vec{K}_{\perp}\right) \delta\left(x_{q}+\Delta x\right)$, of Eq. (19) is the overlap $\langle K \mid P\rangle$. This factor enters into the matrix elements that we compute, but according to the usual Feynman rules does not appear in the final invariant amplitudes. The remaining factors are $\delta^{m n}$ and a dimensionless matter $(M)$ form factor, $F_{M}(q)$ :

$$
\begin{aligned}
F_{M}(q) \equiv & \int \mathrm{d} x_{1} \mathrm{~d} x_{2} \mathrm{~d} x_{3} \delta\left(1-x_{1}-x_{2}-x_{3}\right) \\
& \times \int \frac{\mathrm{d}^{2} p_{1} \mathrm{~d}^{2} p_{2} \mathrm{~d}^{2} p_{3}}{\left(16 \pi^{3}\right)^{2}} \delta\left(\vec{p}_{1}+\vec{p}_{2}+\vec{p}_{3}\right) \\
& \times \sum_{\lambda_{i}} \psi_{3}^{*}\left(k_{1}, k_{2}, k_{3}\right) \psi_{3}\left(p_{1}, p_{2}, p_{3}\right) .
\end{aligned}
$$

If the transverse momentum transfer $\vec{K}_{\perp}$ is also much smaller than the typical momenta of the quarks in the proton, the remaining integral is proportional to the normalization integral for $\psi_{3}$ given in Eq. (4). In that case,

$$
\left\langle K\left|\left[\tilde{\rho}_{q}^{m n}\right]_{1}\right| P\right\rangle \simeq 16 \pi^{3} \delta^{m n} \delta\left(\vec{q}+\vec{K}_{\perp}\right) \delta\left(x_{q}+\Delta x\right) .
$$

Indeed, stripping off the color space identity matrix and setting both $x_{q}$ and $\vec{q}$ to zero leads back to the normalization condition in Eq. (6) for the proton wave function.

\section{LIGHT FRONT EXPECTATION VALUES OF COLOR CHARGE DENSITIES AND FORM FACTORS}

After the prior discussion of the essential preliminaries, we have all the elements in place to construct the light front color charge operator and expectation values of moments of expectation values of this operator in the large $x$ kinematic region where valence quarks dominate. We will later discuss the relation of these correlators to cross sections for exclusive DIS final states.

\section{A. The color charge density operator}

The color charge current density associated with $f=$ $1 \ldots N_{f}$ fermion fields $\psi_{f}$ is $j^{\mu a}=\bar{\psi}_{i, f} \gamma^{\mu} \psi_{j, f}\left(t^{a}\right)_{i j}$. Here, $t^{a}$, $a=1 \ldots 8$ are the generators of the fundamental representation of color-SU(3) normalized as $\operatorname{tr} t^{a} t^{b}=\delta^{a b} / 2$. They are Hermitian and traceless, $\operatorname{tr} t^{a}=0$.

The quark creation and annihilation operators are defined from the Fourier mode expansion of the free field operator at light-cone time $x^{+}=t+z=0$. Since we are focused here on valence quark color charge distributions, we ignore antiquark contributions to write (see Appendix II in [82])

$$
\begin{aligned}
\psi_{i, f}(r) & =\int \frac{\mathrm{d} p^{+} \mathrm{d}^{2} p}{16 \pi^{3} p^{+}} \sum_{s} b_{p, i, s, f} u^{s}(p) e^{-i p \cdot r} \\
& =\int \frac{\mathrm{d} x_{p} \mathrm{~d}^{2} p}{16 \pi^{3} x_{p}} \sum_{s} b_{p, i, s, f} u^{s}(p) e^{-i p \cdot r}
\end{aligned}
$$

where $r \equiv\left(x^{+}=0, x^{-}, \vec{x}_{\perp}\right)$ is the coordinate vector. We wrote out spin and flavor indices explicitly in Eq. (22) and introduced the momentum fraction $x_{p}=p^{+} / P^{+}$. The integration over $p^{+}$or $x_{p}$ is restricted to positive values. Using $\bar{u}_{k} \gamma^{+} u_{p}=2 \sqrt{k^{+} p^{+}}$we can then write the color charge density operator $\rho^{a} \equiv j^{+a}$ as

$$
\begin{aligned}
\rho^{a}(r)= & 2 P^{+} \sum_{\lambda, \lambda^{\prime}} \int \frac{\mathrm{d} x_{q} \mathrm{~d}^{2} q}{16 \pi^{3} \sqrt{x_{q}}} b_{q, i, \lambda}^{\dagger} e^{i q \cdot r} \\
& \times \int \frac{\mathrm{d} x_{p} \mathrm{~d}^{2} p}{16 \pi^{3} \sqrt{x_{p}}} b_{p, j, \lambda^{\prime}} e^{-i p \cdot r}\left(t^{a}\right)_{i j} \delta_{\lambda \lambda^{\prime}} .
\end{aligned}
$$

Note that here $b^{\dagger} b$ is diagonal in spin and flavor, collectively denoted here by $\lambda$. Performing a three-dimensional Fourier transform with respect to $x^{-}$and $\vec{x}$, we obtain the color charge density operator in momentum space,

$$
\tilde{\rho}^{a}\left(x_{k}, \vec{k}\right)=\sum_{\lambda} \int \frac{\mathrm{d} x_{q}}{\sqrt{x_{q}\left(x_{q}+x_{k}\right)}} \int \frac{\mathrm{d}^{2} q}{16 \pi^{3}} b_{q, i, \lambda}^{\dagger} b_{k+q, j, \lambda}\left(t^{a}\right)_{i j} .
$$

In this expression, there is a shift of the argument of the annihilation operator by $\left(k^{+}, \vec{k}\right)=\left(x_{k} P^{+}, \vec{k}\right)$ relative to the quark creation operator. The physical interpretation of $x_{k}$ is that it is the longitudinal momentum shift of the quark momentum following an interaction with a colored probe. In the high energy limit, where $P^{+}$is large, the $x_{k}$ dependent corrections are of order $1 / P^{+}$and can be ignored. This is explained in Appendix A, where we show that the density is confined to a thin pancake in $x^{-}$, with support $1 / P^{+}$. Thus to leading power in $P^{+}$, we approximate [in the notation of Eq. (17)] $\tilde{\rho}^{a}\left(x_{k} \rightarrow 0, \vec{k}\right) \equiv \tilde{\rho}^{a}(\vec{k})$ so that

$$
\begin{aligned}
\tilde{\rho}^{a}(\vec{k}) & =\bigcup_{q, \lambda} b_{x_{q}, \vec{q}, i, \lambda}^{\dagger} b_{x_{q}, \vec{k}+\vec{q}, j, \lambda}\left(t^{a}\right)_{i j} \\
& =\bigcup_{q, \lambda} b_{x_{q}, \vec{q}-\vec{k}, i, \lambda}^{\dagger} b_{x_{q}, \vec{q}, j, \lambda}\left(t^{a}\right)_{i j} .
\end{aligned}
$$

The operator in Eq. (25) differs from that in Eq. (16) because there is no shift in the longitudinal momentum. We use this expression in the remainder of this paper. Note that the variables $\left(x_{q}, \vec{q}\right)$ are integrated over, so that the left-hand side depends only on $\vec{k}$. 

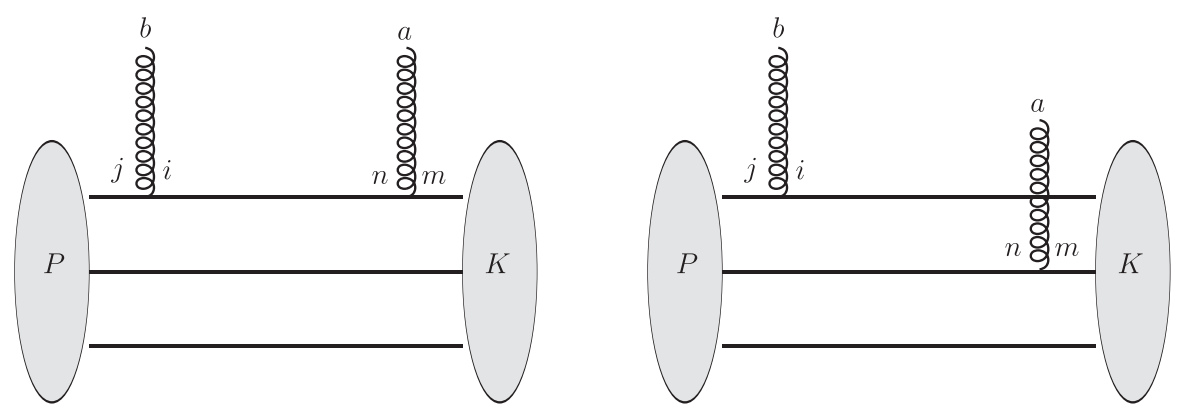

FIG. 1. Illustration of the terms we call one-body (left figure) and two-body (right figure) contribution to the $\left\langle\rho^{a} \rho^{b}\right\rangle$ correlator. $i, j, n$, $m=1,2,3$ denote the colors of the quarks while $a, b=1 \ldots 8$ are those of the gluons that couple to them.

The color charge density per unit transverse area, given by the two-dimensional Fourier transform of this expression, is ${ }^{2}$

$\rho^{a}\left(\vec{x}_{\perp}\right)=\int \frac{\mathrm{d}^{2} k}{(2 \pi)^{2}} e^{i \vec{k} \cdot \vec{x}_{\perp}} \bigcup_{q, \lambda} b_{x_{q}, \vec{q}-\vec{k}, i, \lambda}^{\dagger} b_{x_{q}, \vec{q}, j, \lambda}\left(t^{a}\right)_{i j}$.

In the following subsections, and in the rest of the paper, we will employ an expectation value defined as

$$
\langle\mathbf{O}\rangle_{K_{\perp}}=\frac{\left\langle P^{+}, \vec{K}_{\perp}|\mathbf{O}| P^{+}, \vec{P}_{\perp}=0\right\rangle}{\langle K \mid P\rangle},
$$

where $\mathbf{O}$ denotes a generic operator constituted of products of $\rho^{a}\left(\vec{x}_{\perp}\right)$ defined above, or its two-dimensional Fourier transform $\tilde{\rho}^{a}(\vec{k})$ in Eq. (25). The overlap $\langle K \mid P\rangle$ in Eq. (6) is the standard one given by

$$
\langle K \mid P\rangle=16 \pi^{3} P^{+} \delta\left(K^{+}-P^{+}\right) \delta\left(\vec{K}_{\perp}-\vec{P}_{\perp}\right) .
$$

We shall be interested in the case when $K^{+}=P^{+}$(see Appendix A).

\section{B. $\left\langle\rho^{a}\right\rangle$ in the proton}

The proton matrix element of the color charge density operator Eq. (25) is given by

$\left\langle\tilde{\rho}^{a}(\vec{q})\right\rangle_{K_{\perp}}=\operatorname{tr} t^{a} \sum_{\lambda_{i}} \int \mathrm{d} x_{1} \mathrm{~d} x_{2} \mathrm{~d} x_{3} \delta\left(1-x_{1}-x_{2}-x_{3}\right) \int \frac{\mathrm{d}^{2} p_{1} \mathrm{~d}^{2} p_{2} \mathrm{~d}^{2} p_{3}}{\left(16 \pi^{3}\right)^{2}} \delta\left(\vec{p}_{1}+\vec{p}_{2}+\vec{p}_{3}\right) \psi_{3}^{*}\left(k_{1}, k_{2}, k_{3}\right) \psi_{3}\left(p_{1}, p_{2}, p_{3}\right)$.

Recall that the arguments of $\psi_{3}^{*}$ are given by $k_{i}^{+}=p_{i}^{+} \equiv x_{i} P^{+}, \vec{k}_{1}=\vec{p}_{1}+\left(1-x_{1}\right) \vec{K}_{\perp}, \vec{k}_{2}=\vec{p}_{2}-x_{2} \vec{K}_{\perp}, \vec{k}_{3}=\vec{p}_{3}-x_{3} \vec{K}_{\perp}$.

Since $\operatorname{tr} t^{a}=0$, the above expression is of course zero, as it should be in QCD. Before we move on to consider higher moments of the charge operator, which are nonzero, it is amusing to consider what charge conjugation does to the above expression. $C \tilde{\rho}^{a}(k) C^{-1}$ is given by an expression similar to Eq. (25) with the replacement $t^{a} \rightarrow-\left(t^{a}\right)^{T}=-\left(t^{a}\right)^{*}$. Therefore,

$$
\begin{aligned}
\left\langle C \tilde{\rho}^{a}(\vec{q}) C^{-1}\right\rangle_{K_{\perp}}= & -\left(\operatorname{tr} t^{a}\right)^{*} \sum_{\lambda_{i}} \int \mathrm{d} x_{1} \mathrm{~d} x_{2} \mathrm{~d} x_{3} \delta\left(1-x_{1}-x_{2}-x_{3}\right) \\
& \times \int \frac{\mathrm{d}^{2} p_{1} \mathrm{~d}^{2} p_{2} \mathrm{~d}^{2} p_{3}}{\left(16 \pi^{3}\right)^{2}} \delta\left(\vec{p}_{1}+\vec{p}_{2}+\vec{p}_{3}\right) \psi_{3}^{*}\left(k_{1}, k_{2}, k_{3}\right) \psi_{3}\left(p_{1}, p_{2}, p_{3}\right) .
\end{aligned}
$$

\section{C. $\left\langle\rho^{a} \rho^{b}\right\rangle$ in the proton}

We shall now compute the first nontrivial color charge correlator, the expectation value of $\tilde{\rho}^{a}(q) \tilde{\rho}^{b}(k)$ in the proton. The contributions to its expectation value can be classified, as is common in many-body physics, into one-body and two-body contributions- - these are illustrated in Fig. 1.

\footnotetext{
${ }^{2}$ The color charge density is actually given by $\rho^{a}\left(\vec{x}_{\perp}\right)$ times the coupling constant $g$. However, we prefer to exhibit all factors of $g$ explicitly and we therefore do not introduce a factor of $g$ in the definition of $\rho^{a}\left(\vec{x}_{\perp}\right)$.
}

We begin with the one-body contribution, where both operators act on the same quark, $\left[\tilde{\rho}^{a}(q) \tilde{\rho}^{b}(k)\right]_{1}=\tilde{\rho}^{a}(q) \tilde{\rho}^{b}(k) \otimes \mathbb{1} \otimes \mathbb{1}+$ permutations.

Then using the anticommutation relation Eq. (12), and keeping only the one-body contribution leads to

$$
\begin{aligned}
& b_{x_{\ell_{1}}, \vec{e}_{1}-\vec{q}, i, \lambda}^{\dagger} b_{x_{\ell_{1}}, \vec{e}_{1}, j, \lambda} b_{x_{\ell_{2}}, \vec{e}_{2}-\vec{k}, m, \lambda^{\prime}}^{\dagger} b_{x_{\ell_{2}}, \vec{e}_{2}, n, \lambda^{\prime}} \\
& \quad \rightarrow \delta_{\ell_{1}, \ell_{2}-k}^{j m, \lambda \lambda^{\prime}} b_{x_{\ell_{1}}, \vec{e}_{1}-\vec{q}, i, \lambda}^{\dagger} b_{x_{\ell_{2}}, \vec{e}_{2}, n, \lambda^{\prime}},
\end{aligned}
$$


and further, using the matrix element of $b^{\dagger} b$ given previously in Eq. (14), we get

$\epsilon_{i_{1} i_{2} i_{3}} \epsilon_{j_{1} j_{2} j_{3}}\left\langle p_{1}^{\prime}, i_{1}, \lambda_{1}^{\prime} ; p_{2}^{\prime}, i_{2}, \lambda_{2}^{\prime} ; p_{3}^{\prime}, i_{3}, \lambda_{3}^{\prime}\left|\left[\tilde{\rho}^{a}(q) \tilde{\rho}^{b}(k)\right]_{1}\right| p_{1}, j_{1}, \lambda_{1} ; p_{2}, j_{2}, \lambda_{2} ; p_{3}, j_{3}, \lambda_{3}\right\rangle=3 \delta^{a b} \delta_{p_{1}^{\prime}, p_{1}-q-k}^{\lambda_{1} \lambda_{1}^{\prime}} \delta_{p_{2}^{\prime}, p_{2}}^{\lambda_{2} \lambda_{2}^{\prime}} \delta_{p_{3}^{\prime}, p_{3}}^{\lambda_{3} \lambda_{2}^{\prime}}$.

The symmetry of $\psi_{3}$ under permutations has been used.

We will next compute the two-body contributions to the second moment of the color charge density, where one of the color charge density operators acts on one quark and the other acts on another quark, as illustrated in Fig. 1. Note that the third quark is a spectator in this process:

$$
\left[\tilde{\rho}^{a}(q)\right]_{1}\left[\tilde{\rho}^{b}(k)\right]_{2}=\tilde{\rho}^{a}(q) \otimes \tilde{\rho}^{b}(k) \otimes \mathbb{1}+\text { permutations. }
$$

Its matrix element is evaluated to be

$$
-3 \delta^{a b} \delta_{p_{1}^{\prime}, p_{1}-q}^{\lambda_{1} \lambda_{1}^{\prime}} \delta_{p_{2}^{\prime}, p_{2}-k}^{\lambda_{2} \lambda^{\prime}} \delta_{p_{3}^{\prime}, p_{3}}^{\lambda_{3} \lambda^{\prime}}
$$

This includes a symmetry factor of 2 and another factor of 3 because there are three such identical terms.

Summing over both the one-body and two-body terms, the matrix element of $\tilde{\rho}^{a} \tilde{\rho}^{b}$ between Fock states is given by

$$
\begin{gathered}
\epsilon_{i_{1} i_{2} i_{3}} \epsilon_{j_{1} j_{2} j_{3}}\left\langle p_{1}^{\prime}, i_{1}, \lambda_{1}^{\prime} ; p_{2}^{\prime}, i_{2}, \lambda_{2}^{\prime} ; p_{3}^{\prime}, i_{3}, \lambda_{3}^{\prime}\left|\tilde{\rho}^{a}(q) \tilde{\rho}^{b}(k)\right| p_{1}, j_{1}, \lambda_{1} ; p_{2}, j_{2}, \lambda_{2} ; p_{3}, j_{3}, \lambda_{3}\right\rangle \\
=3 \delta^{a b}\left\{\delta_{p_{1}^{\prime}, p_{1}-q-k}^{\lambda_{1} \lambda_{1}^{\prime}} \delta_{p_{2}^{\prime}, p_{2}}^{\lambda_{2} \lambda_{2}^{\prime}} \delta_{p_{3}^{\prime}, p_{3}}^{\lambda_{3} \lambda_{3}^{\prime}}-\delta_{p_{1}^{\prime}, p_{1}-q}^{\lambda_{1} \lambda_{1}^{\prime}} \delta_{p_{2}^{\prime}, p_{2}-k}^{\lambda_{2} \lambda_{2}^{\prime}} \delta_{p_{3}^{\prime}, p_{3}}^{\lambda_{3} \lambda_{3}^{\prime}}\right\} .
\end{gathered}
$$

As a final step, we need to integrate this expression over the phase-space distribution of the quarks in the proton:

$$
\begin{aligned}
\left\langle\tilde{\rho}^{a}(q) \tilde{\rho}^{b}(k)\right\rangle_{K_{\perp}}= & \frac{1}{2} \delta^{a b} \sum_{\lambda_{i}} \int \mathrm{d} x_{1} \mathrm{~d} x_{2} \mathrm{~d} x_{3} \delta\left(1-x_{1}-x_{2}-x_{3}\right) \int \frac{\mathrm{d}^{2} p_{1} \mathrm{~d}^{2} p_{2} \mathrm{~d}^{2} p_{3}}{\left(16 \pi^{3}\right)^{2}} \delta\left(\vec{p}_{1}+\vec{p}_{2}+\vec{p}_{3}\right)\left[\psi_{3}^{*}\left(k_{1}, k_{2}, k_{3}\right)\right. \\
& \left.-\psi_{3}^{*}\left(\bar{k}_{1}, \bar{k}_{2}, \bar{k}_{3}\right)\right] \psi_{3}\left(p_{1}, p_{2}, p_{3}\right) .
\end{aligned}
$$

The arguments of $\psi_{3}^{*}$ are $k_{i}^{+}=\vec{k}_{i}^{+}=x_{i} P^{+}, \vec{k}_{1}=\vec{p}_{1}+\left(1-x_{1}\right) \vec{K}_{\perp}, \vec{k}_{2}=\vec{p}_{2}-x_{2} \vec{K}_{\perp}, \vec{k}_{3}=\overrightarrow{\vec{k}}_{3}=\vec{p}_{3}-x_{3} \vec{K}_{\perp}, \overrightarrow{\vec{k}}_{1}=\vec{p}_{1}-\vec{q}-x_{1} \vec{K}_{\perp}$, $\overrightarrow{\vec{k}}_{2}=\vec{p}_{2}-\vec{k}-x_{2} \vec{K}_{\perp}$, and all flavors and helicities with $\lambda_{i}^{\prime}=\lambda_{i}$. Note that the rhs does depend on $\vec{q}$ and $\vec{k}$, even at fixed momentum transfer $\vec{K}_{\perp}$, because $\overrightarrow{\vec{k}}_{1}$ and $\overrightarrow{\vec{k}}_{2}$ depend on $\vec{q}, \vec{k}$. The prefactor results from the color algebra. The remaining term is a color charge form factor, $\mathcal{G}$, that contains intrinsically nonperturbative information on the color charge distributions in the three valence quark state of the proton. Thus we rewrite Eq. (37) as

$$
\left\langle\tilde{\rho}^{a}(q) \tilde{\rho}^{b}(k)\right\rangle_{\vec{K}_{\perp}}=\frac{1}{2} \delta^{a b} \mathcal{G}\left(\vec{k}, \vec{K}_{\perp}\right),
$$

with

$$
\begin{gathered}
\mathcal{G}\left(\vec{k}, \vec{K}_{\perp}\right) \equiv \mathcal{G}_{1}\left(\vec{K}_{\perp}\right)-\mathcal{G}_{2}\left(\vec{k}, \vec{K}_{\perp}\right) \\
\mathcal{G}_{1}\left(\vec{K}_{\perp}\right)=\int \mathrm{dPS}_{3} \psi_{3}^{*}\left(p_{1}+\left(1-x_{1}\right) \vec{K}_{\perp}, p_{2}-x_{2} \vec{K}_{\perp}, p_{3}-x_{3} \vec{K}_{\perp}\right) \psi_{3}\left(p_{1}, p_{2}, p_{3}\right) \\
\mathcal{G}_{2}\left(\vec{k}, \vec{K}_{\perp}\right)=\int \mathrm{dPS}_{3} \psi_{3}^{*}\left(p_{1}+\vec{k}+\left(1-x_{1}\right) \vec{K}_{\perp}, p_{2}-\vec{k}-x_{2} \vec{K}_{\perp}, p_{3}-x_{3} \vec{K}_{\perp}\right) \psi_{3}\left(p_{1}, p_{2}, p_{3}\right)
\end{gathered}
$$

The hybrid notation $p_{1}+\left(1-x_{1}\right) \vec{K}_{\perp}$ etc. means that the quantum numbers of $p_{1}$ are unchanged, except that the transverse momentum is increased by $\left(1-x_{1}\right) \vec{K}_{\perp}$. Note further that $\mathrm{PS}_{3}$ is a compact notation for the sum over helicities and momentum phase-space integrals in Eq. (37). 
The form factor $\mathcal{G}$ enters in calculations of the two-gluon exchange model of the Pomeron [84]. Those early authors used simple models in their evaluations. The present formulation is more general and allows for the inclusion of a variety of models; see e.g., [85-91].

For forward scattering, $\vec{K}_{\perp}=0$,

$$
\mathcal{G}(\vec{k}, 0)=1-\mathcal{G}_{2}(\vec{k}, 0)
$$

This quantity vanishes as $|\vec{k}|$ approaches 0 , because $\mathcal{G}_{2}(0,0)=1$, according to the normalization condition for $\psi_{3}$. This vanishing of $\mathcal{G}(\vec{k}, 0)$, caused by the influence of color neutrality, leads to the suppression of infrared divergences.

\section{Relation to the McLerran-Venugopalan (MV) model}

It is worthwhile and interesting to compare our results for the proton with those of the MV model [32-34] approximation, valid for a large nucleus of radius $R$. In the first MV paper [32], $\mu^{2}$ is defined by the relation

$$
\left\langle\rho\left(\vec{x}_{\perp}\right) \rho\left(\vec{y}_{\perp}\right)\right\rangle_{K_{\perp}=0}=\mu^{2} \delta\left(\vec{x}_{\perp}-\vec{y}_{\perp}\right),
$$

where $\mu^{2}$ is the average square of the color charge per unit area. In the original MV model, only the case of zero momentum transfer $K_{\perp}=0$ between the initial and final states of the nucleus was considered. Since $\rho$ has dimensions of inverse area, the state defined by the brackets must have no dimensions.

Later work (see e.g., [92]) showed that $\mu^{2}$ is a function that can depend on $x_{\perp}, y_{\perp}$ and the expression above can be generalized to

$$
\begin{aligned}
& \int d^{2} R_{\perp}\left\langle\rho^{a}\left(\vec{R}_{\perp}+\vec{s}_{\perp} / 2\right) \rho^{b}\left(\vec{R}_{\perp}-\vec{s}_{\perp} / 2\right)\right\rangle_{K_{\perp}=0} \\
& =\delta^{a b} \mu_{\mathrm{MV}}^{2}\left(\vec{s}_{\perp}\right) .
\end{aligned}
$$

Our formulation is in terms of momentum, so here we take the state $|\cdots\rangle$ to be the momentum eigenstate $|P\rangle$ and Fourier transform by operating with $\int d^{2} s_{\perp} e^{-i \vec{k}_{\perp} \cdot \vec{s}_{\perp}}$ on both sides of Eq. (44). The result is

$$
\begin{aligned}
& \delta^{a b} \int d^{2} s_{\perp} e^{-i \vec{k}_{\perp} \cdot \vec{s}_{\perp}} \mu_{\mathrm{MV}}^{2}\left(\vec{s}_{\perp}\right) \\
& \equiv \widetilde{\mu}^{2}{ }_{\mathrm{MV}}\left(\vec{k}_{\perp}\right)=\left\langle\rho^{a}\left(\vec{k}_{\perp}\right) \rho^{b}\left(-\vec{k}_{\perp}\right)\right\rangle_{K_{\perp}=0} .
\end{aligned}
$$

As suggested previously $[93,94]$, and as shown explicitly in [92], imposing a color neutrality condition $\int d^{2} x_{\perp} \rho^{a}\left(x_{\perp}\right)=0$ over a radial distance of $1 / \Lambda$, where $\Lambda$ is a color neutralization scale, gives

$$
\begin{aligned}
& {\widetilde{\mu^{2}}}_{\mathrm{MV}}\left(\vec{k}_{\perp}\right) \rightarrow 0 \text { for } k_{\perp} \rightarrow 0, \quad \text { and } \\
& \widetilde{\mu}^{2} \mathrm{MV}\left(\vec{k}_{\perp}\right)=\mathrm{constant} \text { for } k_{\perp}>\Lambda .
\end{aligned}
$$

In the approach employed here, the use of Eq. (39) and the dimensionless momentum eigenstate leads to the result

$$
\widetilde{\mu^{2}}\left(\overrightarrow{\mathrm{kV}}_{\perp}\right)=\frac{\left(N_{c}^{2}-1\right)}{2}\left(1-\mathcal{G}_{2}\left(\vec{k}_{\perp}\right)\right) .
$$

Just as in Eq. (46), based on the normalization constraint on $\mathcal{G}_{2}\left(\vec{k}_{\perp}\right)$ discussed after Eq. (42), $\widetilde{\mu}^{2}{ }_{\mathrm{MV}}\left(\vec{k}_{\perp}\right)$ vanishes for $k_{\perp} \rightarrow 0$. The structure of $\mathcal{G}_{2}\left(\vec{k}_{\perp}\right)$ in the $K_{\perp}=0$ limit of Eq. (41) suggests on general grounds that it vanishes at large values of $k_{\perp}$. The latter limit corresponds to the MV model [32-34] approximation, valid for a large nucleus:

$$
\frac{1}{2} \mathcal{G}(\vec{q}, 0) \rightarrow \bar{\mu}^{2}{ }_{\mathrm{MV}} \Theta\left(q^{2}-\Lambda^{2}\right) .
$$

Relating Eq. (47) to Eq. (48) allows the identification of the scale $\Lambda$ with a momentum on the order of the inverse of the radius of the proton.

We can apply the formalism computed thus far to compute the gluon distribution of the proton $[28,48,95]$. The number of gluons in the hadron wave function, having longitudinal momenta between $x P^{+}$and $(x+d x) P^{+}$, and a transverse size $\Delta x_{\perp} \sim 1 / Q$, is denoted as $G\left(x, Q^{2}\right) d x$ and is given by

$$
\begin{aligned}
x G\left(x, Q^{2}\right)= & \frac{1}{\pi} \int \frac{d^{2} k_{\perp}}{(2 \pi)^{2}} \Theta\left(Q^{2}-k_{\perp}^{2}\right) \\
& \times\left\langle F_{a}^{i+}\left(\vec{k}_{\perp}\right) F_{a}^{i+}\left(-\vec{k}_{\perp}\right)\right\rangle_{K_{\perp}=0},
\end{aligned}
$$

where $F_{a}^{i+}$ is the color-electric field.

Solving the Yang-Mills equations in the light-cone gauge, to linear order in the color charge density, one obtains [28]

$$
F_{a}^{+i}\left(\vec{k}_{\perp}\right) \simeq i g \frac{k^{i}}{k_{\perp}^{2}} \rho^{a}\left(\vec{k}_{\perp}\right)
$$

and

$$
\left\langle F_{a}^{i+}\left(\vec{k}_{\perp}\right) F_{a}^{i+}\left(-\vec{k}_{\perp}\right)\right\rangle_{K_{\perp}=0} \simeq \frac{g^{2}}{k_{\perp}^{2}}\left\langle\rho^{a}\left(\vec{k}_{\perp}\right) \rho^{a}\left(-\vec{k}_{\perp}\right)\right\rangle_{K_{\perp}=0} .
$$

Inserting this expression into Eq. (49) and using Eq. (39) one obtains the expression

$$
x G\left(x, Q^{2}\right) \simeq \frac{g^{2}}{4 \pi^{2}} \frac{\left(N_{c}^{2}-1\right)}{2} \int_{0}^{Q^{2}} \frac{d k_{\perp}^{2}}{k_{\perp}^{2}}\left(1-\mathcal{G}_{2}\left(\vec{k}_{\perp}, 0\right)\right) .
$$


A comparison of Eq. (52) with the corresponding expression in [96] reaffirms the result in Eq. (47). Note that the integral over $k_{\perp}$ does not have an infrared divergence. As discussed earlier, this is a consequence of the color neutrality of the nucleon. If one breaks up the integral in Eq. (52) into a piece from $0<k_{\perp}<\Lambda$ and another from $\Lambda<k_{\perp}<Q$, the former will integrate to a constant while the latter will give a factor $\frac{\alpha_{S} N_{c}}{\pi} C_{F} \ln \left(Q^{2} / \Lambda^{2}\right)$, where $\alpha_{S}=$ $g^{2} / 4 \pi$ and $C_{F}=\left(N_{c}^{2}-1\right) / 2 N_{c}$ is the Casimir of a quark in the fundamental representation. Thus in the Bjorken limit of $Q^{2} \rightarrow \infty$, one obtains the usual leading contribution [30] to the gluon distribution

$$
x G\left(x, Q^{2}\right) \approx \frac{\alpha_{S} N_{c}}{\pi} C_{F} \ln \left(Q^{2} / \Lambda^{2}\right) .
$$

Interestingly, the effect of color neutralization as imposed on the MV model is also obtained by QCD evolution of the MV model to small $x[96,97]$. Gluons emitted by the quarks screen each other at a saturation scale $Q_{S}(x)$ [37,38]; for small $x, Q_{S}^{2}(x) \gg \Lambda^{2}$. More specifically, $Q_{S}^{2} \propto \mu_{\mathrm{JIMWLK}}^{2}$, where $\mu_{\text {JIMWLK }}^{2}$ is the variance of the Gaussian weight functional for $W[\rho]$ that reproduces the BalitskyJIMWLK hierarchy [50,51] in the CGC EFT. However, while numerical simulations suggest that there is a renormalization group (RG) flow to this Gaussian fixed point [57], it remains an open question at what values of $x$ this is achieved. This concern is in particular germane to the proton, where the color charge densities are not a priori large.

Nevertheless, even if the Gaussian approximation of the CGC EFT is not robust, one can still make considerable progress by computing $\langle\tilde{\rho} \tilde{\rho}\rangle$ from first principles on the light front. Even though our result for $\langle\tilde{\rho} \tilde{\rho}\rangle$ is for the three valence quark state, it is straightforward, with some effort, to extend it to include Fock states containing gluons. A more important issue though is that higher combinants $\left\langle\tilde{\rho}^{a}\left(q_{1}\right) \tilde{\rho}^{b}\left(q_{2}\right) \cdots \tilde{\rho}^{k}\left(q_{n}\right)\right\rangle$ for $n \geq 3$ cannot be expressed in terms of $\langle\tilde{\rho} \tilde{\rho}\rangle$, as they would be if $W[\rho]$ had a Gaussian form.

In our approach, these higher combinants can be computed without invoking a $W[\rho]$ functional at all. These can be computed explicitly and expressed in terms of the corresponding color charge form factors, as in Eq. (39). The latter, as we shall illustrate in subsequent sections, can be extracted from exclusive measurements in DIS at large $x$. Besides our intrinsic interest in the shape and momentum distribution of color charges at large $x$, an important consequence, for the RG discussion above, is a novel strategy whereby one can study systematically the many-body RG flow of these color charge distributions to the putative Gaussian fixed point. To illustrate this strategy, we will compute $\left\langle\rho^{a} \rho^{b} \rho^{c}\right\rangle$ for the three quark valence state and identify the corresponding color charge form factor. This will also have interesting consequences in its own right, which we shall discuss in Sec. IV.

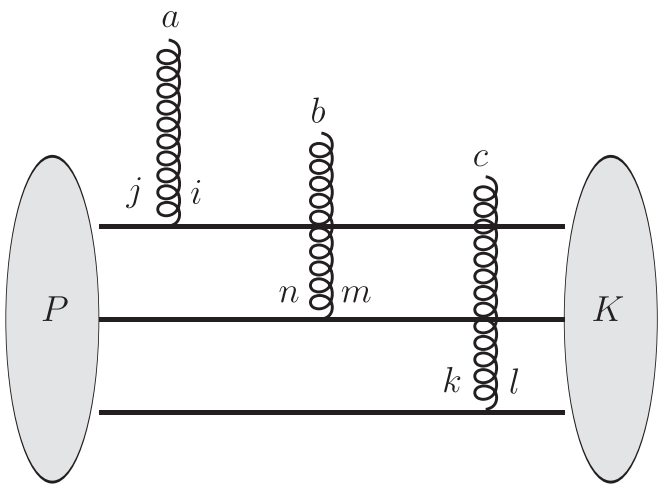

FIG. 2. Illustration of the three-body contribution to the $\left\langle\rho^{a} \rho^{b} \rho^{c}\right\rangle$ correlator.

\section{E. $\left\langle\tilde{\boldsymbol{\rho}}^{a} \tilde{\boldsymbol{\rho}}^{b} \tilde{\boldsymbol{\rho}}^{c}\right\rangle$ in the proton}

To compute the expectation value of $\tilde{\rho}^{a}\left(q_{1}\right) \tilde{\rho}^{b}\left(q_{2}\right) \tilde{\rho}^{c}\left(q_{3}\right)$ in the proton, in addition to the one-body and two-body terms discussed previously, we will have an additional three-body term, which is illustrated in Fig. 2.

\section{One-body contribution}

As previously for $\langle\tilde{\rho} \tilde{\rho}\rangle$, we start with the one-body contribution where all three charge operators act on the same quark. Defining this term as

$$
\begin{aligned}
& {\left[\tilde{\rho}^{a}\left(q_{1}\right) \tilde{\rho}^{b}\left(q_{2}\right) \tilde{\rho}^{c}\left(q_{3}\right)\right]_{1}} \\
& \quad=\tilde{\rho}^{a}\left(q_{1}\right) \tilde{\rho}^{b}\left(q_{2}\right) \tilde{\rho}^{c}\left(q_{3}\right) \otimes \mathbb{1} \otimes \mathbb{1}+\text { permutations },
\end{aligned}
$$

we find

$$
\begin{aligned}
& \left\langle\left[\tilde{\rho}^{a}\left(q_{1}\right) \tilde{\rho}^{b}\left(q_{2}\right) \tilde{\rho}^{c}\left(q_{3}\right)\right]_{1}\right\rangle_{K_{\perp}} \\
& =\operatorname{tr} t^{a} t^{b} t^{c} \int \mathrm{d} x_{1} \mathrm{~d} x_{2} \mathrm{~d} x_{3} \delta\left(1-x_{1}-x_{2}-x_{3}\right) \\
& \quad \times \int \frac{\mathrm{d}^{2} p_{1} \mathrm{~d}^{2} p_{2} \mathrm{~d}^{2} p_{3}}{\left(16 \pi^{3}\right)^{2}} \delta\left(\vec{p}_{1}+\vec{p}_{2}+\vec{p}_{3}\right) \\
& \quad \times \sum_{\lambda_{i}} \psi_{3}^{*}\left(k_{1}, k_{2}, k_{3}\right) \psi_{3}\left(p_{1}, p_{2}, p_{3}\right) .
\end{aligned}
$$

The arguments of $\psi_{3}^{*}$ are $k_{i}^{+}=x_{i} P^{+}, \vec{k}_{1}=\vec{p}_{1}+\left(1-x_{1}\right) \vec{K}_{\perp}$, $\vec{k}_{2}=\vec{p}_{2}-x_{2} \vec{K}_{\perp}, \vec{k}_{3}=\vec{p}_{3}-x_{3} \vec{K}_{\perp}$, and all flavors and helicities are unchanged $\left(\lambda_{i}^{\prime}=\lambda_{i}\right)$. The color factor is given by

$$
\operatorname{tr} t^{a} t^{b} t^{c}=\frac{1}{4} d^{a b c}+\frac{i}{4} f^{a b c} .
$$

Since the $\vec{k}_{i}$ do not explicitly involve the $\vec{q}_{i}$, it follows that at fixed $\vec{K}_{\perp}$, the expectation value of the one-body term is a constant times the delta function constraint on their momentum arguments. 


\section{Two-body contribution}

The computation of the two-body contribution follows analogously to previously. In this case, two of the charge operators act on one quark, while the third $\rho$-operator acts on a second quark. There are three separate terms, corresponding to the three different possible spectator quarks. The first term can be written as

$$
\begin{aligned}
& {\left[\tilde{\rho}^{a}\left(q_{1}\right) \tilde{\rho}^{b}\left(q_{2}\right)\right]_{1}\left[\tilde{\rho}^{c}\left(q_{3}\right)\right]_{2}} \\
& \quad=\tilde{\rho}^{a}\left(q_{1}\right) \tilde{\rho}^{b}\left(q_{2}\right) \otimes \tilde{\rho}^{c}\left(q_{3}\right) \otimes \mathbb{1}+\text { permutations. }
\end{aligned}
$$

We then find

$$
\begin{aligned}
& \left\langle\left[\tilde{\rho}^{a}\left(q_{1}\right) \tilde{\rho}^{b}\left(q_{2}\right)\right]_{1}\left[\tilde{\rho}^{c}\left(q_{3}\right)\right]_{2}\right\rangle_{K_{\perp}} \\
& =-\operatorname{tr} t^{a} t^{b} t^{c} \int \mathrm{d} x_{1} \mathrm{~d} x_{2} \mathrm{~d} x_{3} \delta\left(1-x_{1}-x_{2}-x_{3}\right) \\
& \quad \times \int \frac{\mathrm{d}^{2} p_{1} \mathrm{~d}^{2} p_{2} \mathrm{~d}^{2} p_{3}}{\left(16 \pi^{3}\right)^{2}} \delta\left(\vec{p}_{1}+\vec{p}_{2}+\vec{p}_{3}\right) \\
& \quad \times \sum_{\lambda_{i}} \psi_{3}^{*}\left(k_{1}, k_{2}, k_{3}\right) \psi_{3}\left(p_{1}, p_{2}, p_{3}\right) .
\end{aligned}
$$

Here $k_{i}^{+}=x_{i} P^{+}, \vec{k}_{1}=\vec{p}_{1}+\vec{q}_{3}+\left(1-x_{1}\right) \vec{K}_{\perp}, \vec{k}_{2}=\vec{p}_{2}-\vec{q}_{3}-$ $x_{2} \vec{K}_{\perp}, \vec{k}_{3}=\vec{p}_{3}-x_{3} \vec{K}_{\perp}$. As usual, all flavors and helicities are kept unchanged $\left(\lambda_{i}^{\prime}=\lambda_{i}\right)$. For the other two two-body contributions, one needs to exchange $\vec{q}_{3}$ in $\vec{k}_{1}$ and $\vec{k}_{2}$ by $\vec{q}_{1}$ and $\vec{q}_{2}$, respectively. Moreover, the color factor for the expectation value of $\left[\tilde{\rho}^{a}\left(q_{1}\right) \tilde{\rho}^{c}\left(q_{3}\right)\right]_{1}\left[\tilde{\rho}^{b}\left(q_{2}\right)\right]_{2}$ is $\operatorname{tr} t^{a} t^{c} t^{b}$ instead of $\operatorname{tr} t^{a} t^{b} t^{c}$.

Unlike the one-body contributions, these contributions do depend on $\vec{q}_{i}$, even at fixed $t=-K_{\perp}^{2}$. Note that if one writes $\vec{q}=\vec{q}_{1}+\vec{q}_{2}=-\vec{q}_{3}-\vec{K}_{\perp}$ and $\vec{k}=\vec{q}_{3}$, the phase-space integral in Eq. (58) is identical to the one which appeared in the two-body contribution to $\left\langle\tilde{\rho}^{a}(q) \tilde{\rho}^{b}(k)\right\rangle$ in Eq. (37). This identity can be seen by direct comparison and serves as a check on the computation.

\section{Three-body contribution}

The three-body operator corresponds to each color charge operator acting on separate valence quarks-see Fig. 2. Defining this term as

$$
\begin{aligned}
& {\left[\tilde{\rho}^{a}\left(q_{1}\right) \tilde{\rho}^{b}\left(q_{2}\right) \tilde{\rho}^{c}\left(q_{3}\right)\right]_{3}} \\
& \quad=\tilde{\rho}^{a}\left(q_{1}\right) \otimes \tilde{\rho}^{b}\left(q_{2}\right) \otimes \tilde{\rho}^{c}\left(q_{3}\right)+\text { permutations },
\end{aligned}
$$

we find

$$
\begin{aligned}
& \left\langle\left[\tilde{\rho}^{a}\left(q_{1}\right) \tilde{\rho}^{b}\left(q_{2}\right) \tilde{\rho}^{c}\left(q_{3}\right)\right]_{3}\right\rangle_{K_{\perp}} \\
& =\frac{1}{2} d^{a b c} \int \mathrm{d} x_{1} \mathrm{~d} x_{2} \mathrm{~d} x_{3} \delta\left(1-x_{1}-x_{2}-x_{3}\right) \\
& \quad \times \int \frac{\mathrm{d}^{2} p_{1} \mathrm{~d}^{2} p_{2} \mathrm{~d}^{2} p_{3}}{\left(16 \pi^{3}\right)^{2}} \delta\left(\vec{p}_{1}+\vec{p}_{2}+\vec{p}_{3}\right) \\
& \quad \times \sum_{\lambda_{i}} \psi_{3}^{*}\left(k_{1}, k_{2}, k_{3}\right) \psi_{3}\left(p_{1}, p_{2}, p_{3}\right) .
\end{aligned}
$$

Here, $k_{i}^{+}=x_{i} P^{+}, \vec{k}_{1}=\vec{p}_{1}-\vec{q}_{1}-x_{1} \vec{K}_{\perp}, \vec{k}_{2}=\vec{p}_{2}-\vec{q}_{2}-$ $x_{2} \vec{K}_{\perp}, \vec{k}_{3}=\vec{p}_{3}-\vec{q}_{3}-x_{3} \vec{K}_{\perp}$. As usual, all flavors and helicities are unchanged $\left(\lambda_{i}^{\prime}=\lambda_{i}\right)$. As in the two-body case, this three-body contribution depends on $\vec{q}_{i}$, even at fixed $t=-K_{\perp}^{2}$.

Our net result for $\left\langle\tilde{\rho}^{a}\left(q_{1}\right) \tilde{\rho}^{b}\left(q_{2}\right) \tilde{\rho}^{c}\left(q_{3}\right)\right\rangle$ is the sum of Eqs. (55), (58) (plus the permutations of momenta indicated below that equation), and Eq. (60). Both the symmetric and antisymmetric structure factors, respectively $d^{a b c}$ and $f^{a b c}$, are proportional to color charge form factors. Specifically, we can express the symmetric (S) piece $\mathrm{as}^{3}$

$\left\langle\left[\tilde{\rho}^{a}\left(q_{1}\right) \tilde{\rho}^{b}\left(q_{2}\right) \tilde{\rho}^{c}\left(q_{3}\right)\right]_{\mathrm{S}}\right\rangle_{K_{\perp}} \equiv \frac{d^{a b c}}{N_{c}} \mathcal{G}_{O}\left(\vec{q}_{1}, \vec{q}_{2}, \vec{q}_{3} ; \vec{K}_{\perp}\right)$,

which involves the one-, two- and three-body terms. Anticipating results to appear, we denote $\mathcal{G}_{O}$ to be the odderon form factor.

We note that similar form factors were discussed previously in the context of high energy forward scattering amplitudes [99,100]. Fukugita and Kwiecinski [99] similarly identified one-body, two-body and three-body contributions and noted that the two-body contribution can be expressed in terms of the Pomeron form factor in Eq. (39). However, though they suggest that the three-body contribution in Eq. (60) can be expressed in terms of the twobody contribution, our results show that this is not true in general. Furthermore, unlike these works, we are able to express our results explicitly in terms of the QCD valence Fock state wave function.

We can however confirm the observation in [100] that in the limit that any of the $\vec{q}_{i} \rightarrow 0$, the sum of all these contributions should vanish. Specifically, taking $\vec{q}_{3} \rightarrow 0$ (but $\vec{q}_{1}, \vec{q}_{2}, \vec{K}_{\perp}$ arbitrary), one observes that the sum of the $a$-, $b$-, and $c$-symmetric pieces of Eqs. (55), (58) and (60) does indeed vanish. The underlying reason is a general feature of QCD that must be satisfied by any model: a long wavelength gluon cannot couple to a color singlet.

\footnotetext{
${ }^{3}$ We introduce an explicit factor of $1 / N_{c}$ on the right-hand side in order to match powers of $N_{c}$ in the odderon amplitude to a computation in perturbative QCD [98]; see below.
} 


\section{COLOR CHARGE FORM FACTORS AND EXCLUSIVE HEAVY QUARK PRODUCTION IN DIS}

In the previous section, we derived explicit expressions for the expectation values of quadratic and cubic combinants of the color charge density and reexpressed the results in terms of nonperturbative color charge form factors. We show here that these nonperturbative quantities can be determined from exclusive measurements of heavy quarkonia in DIS at large $x$ at Jefferson Laboratory [101-103] and in the future at the Electron-Ion Collider [104]. We derive the amplitude for exclusive quarkonium production and express it in terms of our Pomeron and odderon color charge form factors in the first subsection. Specifically, we show that the exclusive $J / \Psi$ cross section is proportional to both the Pomeron and odderon form factors. In contrast, the $\eta_{c}$ amplitude depends only on the odderon form factor; the latter can therefore be extracted directly from an exclusive measurement of the production of $\eta_{c}$ mesons. While this possibility is well known in the literature, and even

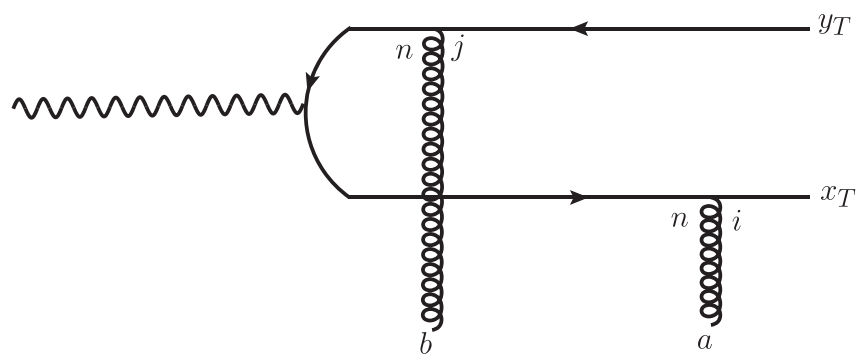

FIG. 3. Illustration of the two-gluon contribution to the DIS exclusive amplitude for $c \bar{c}$ production.

discussed very recently [105], we will articulate how our work brings a novel perspective to this discussion.

\section{A. Amplitude for exclusive quarkonium production at large $\boldsymbol{x}_{\mathrm{Bj}}$}

In DIS at high energies, the amplitude for exclusive quarkonium production be expressed as [106]

$$
\mathcal{A}^{\gamma^{*} p \rightarrow Q \bar{Q} p}\left(Q^{2}, \vec{K}_{\perp}\right) \sim i \int \mathrm{d}^{2} r \int_{0}^{1} \frac{d z}{4 \pi}\left(\Psi_{\gamma^{*}} \Psi_{Q \bar{Q}}^{*}\right)\left(\vec{r}, z, Q^{2}\right) e^{-i \frac{(1-2 z)}{2} \vec{r} \cdot \vec{K}_{\perp}} \int d^{2} b_{\perp} e^{i \vec{b}_{\perp} \cdot \vec{K}_{\perp}} \mathcal{T}\left(\vec{r}, \vec{b}_{\perp} ; \vec{K}_{\perp}\right)
$$

Here $\Psi_{\gamma^{*}}$ is the light-cone wave function of a virtual photon to fluctuate into a charm-anticharm pair [107] of relative size $\vec{r}, z(1-z)$ is the fraction of the photon momentum taken by the quark (antiquark) and $\vec{K}_{\perp}$ is the transverse momentum transfer between the incoming and outgoing proton. Further, $\Psi_{Q \bar{Q}}\left(\vec{r}, z, Q^{2}\right)$ is the wave function corresponding to the overlap $\langle c \bar{c} \mid Q \bar{Q}\rangle$ of the $c \bar{c}$ pair with any $Q \bar{Q}$ quarkonium state $\left[J / \Psi, \Psi(2 S), \eta_{c}, \chi_{c}, \ldots\right]$.

Finally, $\mathcal{T}$ denotes the invariant amplitude for elastic scattering of the $c \bar{c}$ pair off color fields in the target proton ${ }^{4}$ and can be expressed as ${ }^{5}[23,108,109]$

$$
\begin{aligned}
& \mathcal{T}\left(\vec{r}, \vec{b}_{\perp} ; \vec{K}_{\perp}\right) \\
& \quad=2 N_{c}\left[1-\frac{1}{N_{c}} \operatorname{tr}\left\langle U\left(\vec{b}_{\perp}+\frac{\vec{r}}{2}\right) U^{\dagger}\left(\vec{b}_{\perp}-\frac{\vec{r}}{2}\right)\right\rangle_{\vec{K}_{\perp}}\right] .
\end{aligned}
$$

Here $U$ (and $U^{\dagger}$ ) are lightlike Wilson lines representing the color rotation of a color dipole in the gauge field background of the proton. The brackets $\langle\cdots\rangle_{K_{\perp}}$ represent taking the expectation value in the proton according to Eq. (27). As in the discussion there, and discussed further in

\footnotetext{
${ }^{4}$ We use the shorthand $\int_{x_{T}} \equiv \int \mathrm{d}^{2} x_{T}$ while $\int_{q} \equiv \int \frac{\mathrm{d}^{2} q}{(2 \pi)^{2}}$.

${ }^{5}$ In [106], the factor of $N_{c}$ is absorbed in the definitions of $\Psi_{\gamma^{*}}$ and $\Psi_{Q \bar{Q}}\left(r, z, Q^{2}\right)$; we feel it is more appropriate to not do so and to keep it explicit in $\mathcal{T}$. To avoid double counting, this should be taken into account while using Eq. (62).
}

Appendix A, we are making an eikonal approximation that the proton target has a large $P^{+}$momentum. In writing Eq. (63), we identified the coordinates $\vec{x}_{T}$ and $\vec{y}_{T}$ of the quark-antiquark pair shown in Fig. 3 with the impact parameter of the quark-antiquark pair and their relative separation respectively as [110]

$$
\begin{aligned}
\overrightarrow{\tilde{b}}_{\perp} & =z \vec{x}_{\perp}+(1-z) \vec{y}_{\perp}, \\
\vec{r} & =\vec{x}_{\perp}-\vec{y}_{\perp},
\end{aligned}
$$

and then a further transformation [108]

$$
\vec{b}_{\perp}=\overrightarrow{\tilde{b}}_{\perp}+\left(\frac{1}{2}-z\right) \vec{r}
$$

to express the result in the symmetric form shown in Eq. (63). The phase factor $e^{-i \frac{(1-2 z)}{2} \cdot \vec{r} \vec{K}_{\perp}}$ in Eq. (62) is a consequence of these transformations.

In Lorenz gauge $\partial_{\mu} A^{\mu}=0$, and in the above described eikonal approximation, the gauge fields appearing in the Wilson lines corresponding to multiple scattering of a quark at spatial position $\left(x^{-}, \vec{x}_{T}\right)$ have only one component $A^{+}$, which satisfies the Poisson equation $\nabla_{\perp}^{2} A^{+}=$ $g \rho\left(x^{-}, \vec{x}_{\perp}\right)\left(A_{\mu} \equiv t^{a} A_{\mu}^{a}\right)$ and the lightlike Wilson lines are path ordered in the $x^{-}$direction $[28,30]$ :

$$
U^{\dagger}\left(\vec{x}_{T}\right)=\mathcal{P} e^{i g \int d x^{-} A^{+}\left(x^{-}, \vec{x}_{T}\right)}
$$




$$
\begin{aligned}
= & 1+i g \int d x^{-} A^{+}\left(x^{-}, \vec{x}_{T}\right)+(i g)^{2} \int d x^{-} \int x^{x^{-}} d y^{-} A^{+}\left(x^{-}, \vec{x}_{T}\right) A^{+}\left(y^{-}, \vec{x}_{T}\right) \\
& +(i g)^{3} \int d x^{-} \int^{x^{-}} d y^{-} \int^{y^{-}} d z^{-} A^{+}\left(x^{-}, \vec{x}_{T}\right) A^{+}\left(y^{-}, \vec{x}_{T}\right) A^{+}\left(z^{-}, \vec{x}_{T}\right)+\cdots,
\end{aligned}
$$

where the factors of $g t^{a}$ contained in this expansion correspond to the vertices arising from the order by order expansion of the coherent coupling of the gluon fields in the target to the $c$ or $\bar{c}$ quark.

Expanding $U\left(\vec{x}_{T}\right) U^{\dagger}\left(\vec{y}_{T}\right)-1$ to third order in $g A^{+}$gives

$$
\begin{aligned}
1-U\left(\vec{x}_{T}\right) U^{\dagger}\left(\vec{y}_{T}\right)= & (i g)^{2} \int d x^{-} \int d y^{-} A^{+}\left(x^{-}, \vec{x}_{T}\right) A^{+}\left(y^{-}, \vec{y}_{T}\right)-(-i g)^{2} \int d x^{-} \int_{x^{-}} d y^{-} A^{+}\left(x^{-}, \vec{x}_{T}\right) A^{+}\left(y^{-}, \vec{x}_{T}\right) \\
& -(i g)^{2} \int d x^{-} \int x^{x^{-}} d y^{-} A^{+}\left(x^{-}, \vec{y}_{T}\right) A^{+}\left(y^{-}, \vec{y}_{T}\right) \\
& -(-i g)(i g)^{2} \int d x^{-} \int d y^{-} \int^{y^{-}} d z^{-} A^{+}\left(x^{-}, \vec{x}_{T}\right) A^{+}\left(y^{-}, \vec{y}_{T}\right) A^{+}\left(z^{-}, \vec{y}_{T}\right) \\
& -(-i g)^{2}(i g) \int d x^{-} \int d y^{-} \int d z^{-} A^{+}\left(x^{-}, \vec{x}_{T}\right) A^{+}\left(y^{-}, \vec{x}_{T}\right) A^{+}\left(z^{-}, \vec{y}_{T}\right) \\
& -(-i g)^{3} \int d x^{-} \int_{x^{-}} d y^{-} \int_{y^{-}} d z^{-} A^{+}\left(x^{-}, \vec{x}_{T}\right) A^{+}\left(y^{-}, \vec{x}_{T}\right) A^{+}\left(z^{-}, \vec{x}_{T}\right) \\
& -(i g)^{3} \int d x^{-} \int^{x^{-}} d y^{-} \int^{y^{-}} d z^{-} A^{+}\left(x^{-}, \vec{y}_{T}\right) A^{+}\left(y^{-}, \vec{y}_{T}\right) A^{+}\left(z^{-}, \vec{y}_{T}\right)+\cdots
\end{aligned}
$$

Let us first consider the expectation value of the previous expression up to order $\left(g A^{+}\right)^{2}$. Using the fact that it is symmetric under $x^{-}-y^{-} \rightarrow y^{-}-x^{-}$, we can express the term appearing in Eq. (63) as

$$
\begin{aligned}
1-\left\langle U\left(\vec{b}_{\perp}+\frac{\vec{r}}{2}\right) U^{\dagger}\left(\vec{b}_{\perp}-\frac{\vec{r}}{2}\right)\right\rangle_{K_{\perp}}= & -g^{2} \int d x^{-} \int d y^{-}\left\langle A^{+}\left(x^{-}, \vec{b}_{\perp}+\frac{\vec{r}}{2}\right) A^{+}\left(y^{-}, \vec{b}_{\perp}-\frac{\vec{r}}{2}\right)\right\rangle_{K_{\perp}} \\
& +\frac{1}{2} g^{2} \int d x^{-} \int d y^{-}\left\langle A^{+}\left(x^{-}, \vec{b}_{\perp}+\frac{\vec{r}}{2}\right) A^{+}\left(y^{-}, \vec{b}_{\perp}+\frac{\vec{r}}{2}\right)\right\rangle_{K_{\perp}} \\
& +\frac{1}{2} g^{2} \int d x^{-} \int d y^{-}\left\langle A^{+}\left(x^{-}, \vec{b}_{\perp}-\frac{\vec{r}}{2}\right) A^{+}\left(y^{-}, \vec{b}_{\perp}-\frac{\vec{r}}{2}\right)\right\rangle_{K_{\perp}} .
\end{aligned}
$$

We can use the Poisson equation to relate $A^{+}$to the charge density operator $\rho$ and further, to write the latter in terms of its two-dimensional Fourier representation. In doing so, note that the integral of $\tilde{\rho}\left(x^{-}, \vec{q}\right)$ over $x^{-}$corresponds to the operator $\tilde{\rho}(q)$ in Eq. (25). We then obtain, to quadratic order in $A^{+}$or $\rho$,

$$
\begin{aligned}
\left.1-\frac{1}{N_{c}} \operatorname{tr}\left\langle U\left(\vec{b}_{\perp}+\frac{\vec{r}}{2}\right)\right) U^{\dagger}\left(\vec{b}_{\perp}-\frac{\vec{r}}{2}\right)\right\rangle_{\vec{K}_{\perp}}^{O\left(\rho^{2}\right)}= & -\frac{g^{4}}{2 N_{c}} \delta^{a b} \int_{q_{1}} \int_{q_{2}} \frac{e^{i \vec{b}_{\perp} \cdot\left(\vec{q}_{1}+\vec{q}_{2}\right)}}{q_{1}^{2} q_{2}^{2}}\left[e^{i \frac{\vec{r} \cdot}{2} \cdot\left(\vec{q}_{1}-\vec{q}_{2}\right)}-\frac{1}{2} e^{i\left(q_{1}+q_{2}\right) \cdot \frac{\vec{r}}{2}}-\frac{1}{2} e^{-i\left(q_{1}+q_{2}\right) \cdot \frac{\vec{r}}{2}}\right] \\
& \times\left\langle\tilde{\rho}^{a}\left(\vec{q}_{1}\right) \tilde{\rho}^{b}\left(\vec{q}_{2}\right)\right\rangle_{\vec{K}_{\perp}} .
\end{aligned}
$$

Multiplying both the lhs and rhs by $2 N_{c}$ to obtain $\mathcal{T}^{O\left(\rho^{2}\right)}$, we can then perform the integration over the impact parameter in Eq. (62) to obtain

$$
\int d^{2} b_{\perp} e^{i \vec{b}_{\perp} \cdot \vec{K}_{\perp}} \mathcal{T}^{O\left(\rho^{2}\right)}\left(\vec{r}, \vec{b}_{\perp} ; \vec{K}_{\perp}\right)=2 N_{c}\left[-\frac{g^{4}}{2 N_{c}} \int_{q_{1}} \frac{1}{q_{1}^{2}\left(\vec{q}_{1}+\vec{K}_{\perp}\right)^{2}}\left(e^{i \frac{\vec{r}}{2} \cdot\left(2 \vec{q}_{1}+\vec{K}_{\perp}\right)}-\cos \left(\frac{\vec{r} \cdot \vec{K}_{\perp}}{2}\right)\right)\left\langle\tilde{\rho}^{a}\left(\vec{q}_{1}\right) \tilde{\rho}^{a}\left(-\vec{q}_{1}-\vec{K}_{\perp}\right)\right\rangle_{K_{\perp}}\right]
$$


Defining the lhs of the above expression to be the Pomeron amplitude $\mathcal{P}\left(\vec{r}, \vec{K}_{\perp}\right)$ and replacing $\left\langle\tilde{\rho}\left(\vec{q}_{1}\right) \tilde{\rho}\left(-\vec{q}_{1}-\vec{K}_{\perp}\right)\right\rangle_{\vec{K}_{T}}$ on the rhs by the Pomeron form factor in Eq. (38), we obtain ${ }^{6}$

$$
\mathcal{P}\left(\vec{r}, \vec{K}_{\perp}\right)=2 N_{c}\left[-\frac{g^{4} C_{F}}{2} \int_{q_{1}} \frac{1}{q_{1}^{2}\left(\vec{q}_{1}+\vec{K}_{\perp}\right)^{2}}\left(e^{i \overrightarrow{\vec{r}_{2}}\left(2 \vec{q}_{1}+\vec{K}_{\perp}\right)}-\cos \left(\frac{\vec{r} \cdot \vec{K}_{\perp}}{2}\right)\right) \mathcal{G}\left(\vec{q}_{1},-\vec{q}_{1}-\vec{K}_{\perp}\right)\right] .
$$

Here $C_{F}=\left(N_{c}^{2}-1\right) / 2 N_{c}$ is the quadratic Casimir in the fundamental representation.

The amplitude for exclusive quarkonium production in DIS can also receive a contribution from three-gluon exchange, as illustrated in Fig. 4. This contribution is recovered in our approach by expanding Eq. (63) to $O\left(\rho^{3}\right)$. We begin by formally rewriting

$$
\begin{aligned}
\mathcal{T}\left(\vec{r}, \vec{b}_{\perp} ; \vec{K}_{\perp}\right)= & 2 N_{c}\left[1-\frac{1}{2 N_{c}} \operatorname{tr}\left(\left\langle U\left(\vec{b}_{\perp}+\frac{\vec{r}}{2}\right) U^{\dagger}\left(\vec{b}_{\perp}-\frac{\vec{r}}{2}\right)\right\rangle_{\vec{K}_{\perp}}+\left\langle U\left(\vec{b}_{\perp}-\frac{\vec{r}}{2}\right) U^{\dagger}\left(\vec{b}_{\perp}+\frac{\vec{r}}{2}\right)\right\rangle_{\vec{K}_{\perp}}\right)\right. \\
& \left.-\frac{1}{2 N_{c}} \operatorname{tr}\left(\left\langle U\left(\vec{b}_{\perp}+\frac{\vec{r}}{2}\right) U^{\dagger}\left(\vec{b}_{\perp}-\frac{\vec{r}}{2}\right)\right\rangle_{\vec{K}_{\perp}}-\left\langle U\left(\vec{b}_{\perp}-\frac{\vec{r}}{2}\right) U^{\dagger}\left(\vec{b}_{\perp}+\frac{\vec{r}}{2}\right)\right\rangle_{\vec{K}_{\perp}}\right)\right]
\end{aligned}
$$

as the sum of a piece that is symmetric under $\vec{b}_{\perp}+\frac{\vec{r}}{2} \leftrightarrow \vec{b}_{\perp}-\frac{\vec{r}}{2}$ and a piece that is antisymmetric under this exchange. Expanding out both the symmetric and antisymmetric terms to $O\left(\left(g A^{+}\right)^{3}\right)$, or equivalently $O\left(\rho^{3}\right)$, we find that the symmetric piece is identically zero at this order. In other words, it is impossible to have color-singlet three-gluon exchange that is even under parity. The contribution of the surviving term can be expressed as the odderon amplitude

$$
i \mathcal{O}\left(\vec{r}, \vec{K}_{\perp}\right)=\int d^{2} b_{\perp} e^{i \vec{b}_{\perp} \cdot \vec{K}_{\perp}} \mathcal{T}^{O\left(\rho^{3}\right)}\left(\vec{r}, \vec{b}_{\perp} ; \vec{K}_{\perp}\right)
$$

where

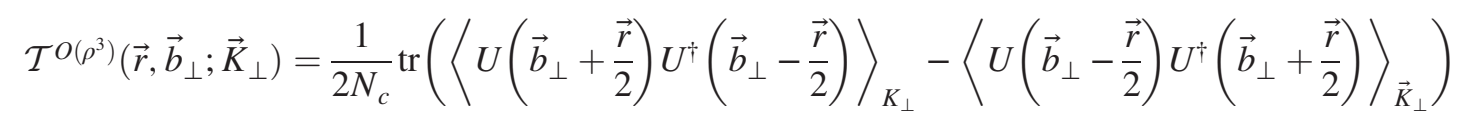

has the form of the expectation value of the odderon operator [111].

Working the rhs out to cubic order in $g A^{+}$(or equivalently $\rho$; see Appendix B for details) one obtains

$$
\begin{aligned}
\mathcal{T}^{O\left(\rho^{3}\right)}\left(\vec{r}, \vec{b}_{\perp} ; \vec{K}_{\perp}\right)= & -\frac{g^{6}}{8 N_{c}} d^{a b c} \int_{q_{1}} \int_{q_{2}} \int_{q_{3}} \frac{1}{q_{1}^{2}} \frac{1}{q_{2}^{2}} \frac{1}{q_{3}^{2}}\left\langle\tilde{\rho}^{a}\left(\vec{q}_{1}\right) \tilde{\rho}^{b}\left(\vec{q}_{2}\right) \tilde{\rho}^{c}\left(\vec{q}_{3}\right)\right\rangle_{K_{\perp}} e^{i \vec{b}_{\perp} \cdot\left(\vec{q}_{1}+\vec{q}_{2}+\vec{q}_{3}\right)} \\
& \times\left[2 \sin \left(\frac{\vec{r}}{2} \cdot\left(\vec{q}_{1}-\vec{q}_{2}-\vec{q}_{3}\right)\right)+\frac{2}{3} \sin \left(\frac{\vec{r}}{2} \cdot\left(\vec{q}_{1}+\vec{q}_{2}+\vec{q}_{3}\right)\right)\right] .
\end{aligned}
$$

Note that only the terms proportional to $\sim d^{a b c}$ from $\left\langle\rho^{a}\left(\vec{q}_{1}\right) \rho^{b}\left(\vec{q}_{2}\right) \rho^{c}\left(\vec{q}_{3}\right)\right\rangle_{K_{\perp}}$ contribute. Further, employing our definition of the odderon amplitude in Eq. (61), and using the identity

$$
d^{a b c} d^{a b e}=\frac{N_{c}^{2}-4}{N_{c}} \delta^{c e},
$$

we obtain the odderon amplitude to be

$$
\begin{aligned}
i \mathcal{O}\left(\vec{r} ; \vec{K}_{\perp}\right)= & -g^{6} \frac{\left(N_{c}^{2}-4\right)\left(N_{c}^{2}-1\right)}{8 N_{c}^{3}} \int_{q_{1}} \int_{q_{2}} \frac{1}{q_{1}^{2}} \frac{1}{q_{2}^{2}} \frac{1}{\left(\vec{q}_{1}+\vec{q}_{2}+\vec{K}_{\perp}\right)^{2}} \mathcal{G}_{O}\left(\vec{q}_{1}, \vec{q}_{2},-\vec{K}_{\perp}-\vec{q}_{1}-\vec{q}_{2} ; \vec{K}_{\perp}\right) \\
& \times\left[2 \sin \left(\frac{\vec{r}}{2} \cdot\left(2 \vec{q}_{1}+\vec{K}_{\perp}\right)\right)-\frac{2}{3} \sin \left(\frac{\vec{r}}{2} \cdot \vec{K}_{\perp}\right)\right],
\end{aligned}
$$

\footnotetext{
${ }^{6}$ In the forward scattering $\vec{K}_{T} \rightarrow 0$ limit, replacing $\frac{1}{2} \mathcal{G}(\vec{q}, 0) \rightarrow \bar{\mu}_{\mathrm{MV}}^{2} \Theta\left(q^{2}-\Lambda^{2}\right)$, as discussed previously, reproduces the MV model expression

$$
\left\langle 1-U\left(\vec{x}_{T}\right) U^{\dagger}\left(\vec{y}_{T}\right)\right\rangle_{K_{\perp}=0}=\frac{g^{4} C_{F}}{2} \int_{q} \frac{1}{q^{4}}\left[e^{i \vec{q} \cdot \vec{r}}-1\right] \bar{\mu}_{\mathrm{MV}}^{2} \Theta\left(q^{2}-\Lambda^{2}\right)+\cdots
$$




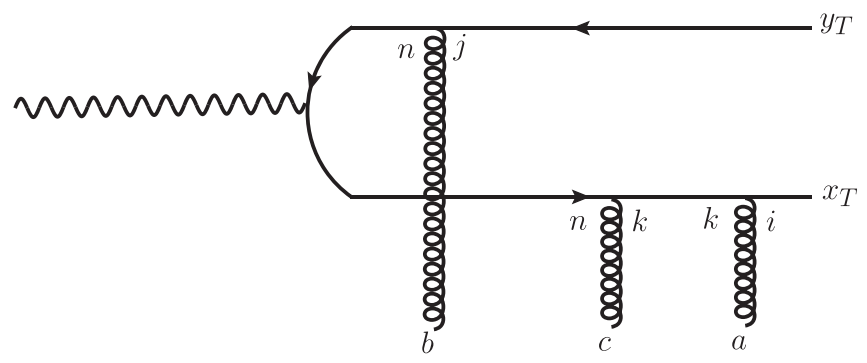

FIG. 4. Illustration of the three-gluon contribution to the DIS exclusive amplitude for $c \bar{c}$ production.

where $\mathcal{G}_{O}\left(\vec{q}_{1}, \vec{q}_{2},-\vec{K}_{\perp}-\vec{q}_{1}-\vec{q}_{2} ; \vec{K}_{\perp}\right)$ is the odderon form factor from Eq. (61) and $\left(N_{c}^{2}-1\right)\left(N_{c}^{2}-4\right) / 4 N_{c}^{2}=C_{3 F}$ is the cubic Casimir constant of $\mathrm{SU}\left(N_{c}\right)$ in the fundamental representation.

The odderon expectation value $i O\left(\vec{x}_{T}, \vec{y}_{T} ; K_{T}=0\right)$ in the forward limit has been computed previously in the MV model, where the weight functional (appropriately normalized) describing the distribution of color charges in a large nucleus has the general form $[36,112]^{7}$

$$
\begin{aligned}
W[\rho]= & \int[d \rho] \exp \left(-\int d^{2} x_{\perp}\left[\frac{\rho^{a}\left(\vec{x}_{\perp}\right) \rho^{a}\left(\vec{x}_{\perp}\right)}{2 \mu^{2}}\right.\right. \\
& \left.\left.-\frac{d_{a b c} \rho^{a}\left(\vec{x}_{\perp}\right) \rho^{b}\left(\vec{x}_{\perp}\right) \rho^{c}\left(\vec{x}_{\perp}\right)}{\kappa_{A}}\right]\right) .
\end{aligned}
$$

The cubic Casimir term here has the weight $\kappa_{A}=$ $g^{3} A^{2} N_{c} / \pi^{2} R^{4}$ and will of course give a nonzero value for the odderon form factor. For a large nucleus, if the typical magnitude of $\rho \sim \sqrt{\mu_{\mathrm{MV}}^{2}} \sim A^{1 / 6}$, this cubic odderon term is subleading relative to the quadratic Pomeron term in $W[\rho]$ by $A^{-1 / 6}$, which is a weak suppression factor even for a large nucleus. The expectation value $i O\left(x_{T}, y_{T}\right)$ of the odderon operator computed in the MV model gives

$i O\left(\vec{x}_{T}, \vec{y}_{T}\right)=\alpha_{S}^{3} \frac{\left(N_{c}^{2}-4\right)\left(N_{c}^{2}-1\right)}{4 \pi r_{0}^{2} N_{c}^{3}} A^{1 / 3} \int d^{2} \mathbf{u} \ln ^{3} \frac{|\mathbf{x}-\mathbf{u}|}{|\mathbf{y}-\mathbf{u}|}$,

where $r_{0}=1.12 \mathrm{fm}$. This expression is also recovered in a perturbative QCD computation [98]. We can compare this expression to Eq. (79), for $A \rightarrow 1$ and in the forward limit of $\vec{K}_{T} \rightarrow 0$. As discussed in [112], the logarithm above can be expressed in terms of the Coulomb propagator in two dimensions. Making use of this fact, we observe that Eq. (81) can be reexpressed as Eq. (79) if the odderon form factor $\mathcal{G}_{O}$ is a constant everywhere except in the infrared due to the previously discussed constraint from color neutrality. Conversely, the structure of $\mathcal{G}_{O}$ in Eq. (79),

${ }^{7}$ A quartic term $\sim \rho^{a}\left(\vec{x}_{\perp}\right) \rho^{a}\left(\vec{x}_{\perp}\right) \rho^{b}\left(\vec{x}_{\perp}\right) \rho^{b}\left(\vec{x}_{\perp}\right) / \kappa_{4}$ arises too [113]; it ensures that the action for $\rho$ is bounded from below. and hence the odderon operator at large $x_{\mathrm{Bj}}$, can be very different from the expectation from the MV model.

\section{B. Cross section for exclusive production of $J / \Psi$ and $\eta_{c}$ mesons at large $x_{\mathrm{Bj}}$}

The general formalism for exclusive quarkonium production that we outlined in the previous section can now be adapted to compute the cross section for specific quarkonium states. We will consider here the $J / \Psi$ because it is the most easily accessible quarkonium state, and the $\eta_{c}$ because it is the lightest state with unique features that promise novel insight into nonperturbative QCD. Since we are interested in manybody color charge correlators of valence Fock states in this work, our discussion is most relevant for exclusive production of these quarkonium states at large $x_{\mathrm{Bj}}$. As noted, this is a regime that is already accessible with the high luminosity DIS experiments at Jefferson Lab and at a future EIC.

The cross section for exclusive $J / \Psi$ production can be expressed as

$$
\frac{d \sigma_{T, L}^{\gamma^{*} p \rightarrow J / \Psi p}}{d t}=\frac{1}{16 \pi}\left|\mathcal{A}_{T, L}^{\gamma^{*} p \rightarrow J / \Psi p}\right|^{2},
$$

where

$$
\begin{aligned}
& \mathcal{A}_{T, L}^{\gamma^{*} p \rightarrow J / \Psi p}\left(Q^{2}, \vec{K}_{\perp}\right) \\
& \sim i \int \mathrm{d}^{2} r \int \frac{d z}{4 \pi}\left(\Psi_{\gamma^{*}} \Psi_{J / \Psi}^{*}\right)\left(\vec{r}, z, Q^{2}\right) \\
& \quad \times e^{-i \frac{(1-2 z)}{2} \vec{r} \cdot \vec{K}_{\perp}}\left[\mathcal{P}\left(\vec{r}, \vec{K}_{\perp}\right)+i \mathcal{O}\left(\vec{r}, \vec{K}_{\perp}\right)\right] .
\end{aligned}
$$

Here $K_{\perp}^{2}=-t$, and $\Psi_{J / \Psi}, \Psi_{\gamma^{*}}$ denote the $J / \psi$ and virtual photon light-cone wave functions (for longitudinal or transverse polarization); their product is summed over the helicities of the $c$ and $\bar{c}$ quarks. Further, $\mathcal{P}$ is the Pomeron contribution to the exclusive $J / \Psi$ amplitude given in Eq. (73) and $i \mathcal{O}$ is the respective odderon contribution given by Eq. (79). The former is directly proportional to the Pomeron color charge form factor and the latter to the odderon color charge form factor. These two terms in $\mathcal{A}_{T, L}^{\gamma^{*} p \rightarrow J / \Psi p}$ contain the important QCD physics underlying the Regge-theory-based descriptions of elastic/exclusive cross sections in terms of imaginary and real terms respectively [114]. There is an additional kinematic contribution coming from the nonzero values of $\Delta x$ discussed in Sec. II; however, as we demonstrate in Appendix A, these contributions are $1 / P^{+}$suppressed.

Some remarks on the contribution due to the odderon are in order. $i \mathcal{O}$ is odd under charge conjugation, which corresponds to the simultaneous transformations $\vec{r} \rightarrow-\vec{r}$, $z \rightarrow 1-z$. On the other hand, $\Psi_{\gamma^{*}} \Psi_{J / \Psi}^{*}$ has even C parity. Therefore, the integral over $i \mathcal{O}$ in Eq. (83) is nonzero only if the final state is restricted to, e.g., $p_{c}^{+}<p_{\bar{c}}^{+}(z<1 / 2)$. This prevents the cancellation of the amplitude with its $\mathrm{C}$ conjugate. Likewise, the odderon contribution to the above 
amplitude will not cancel against its parity transform if the direction of the momentum transfer $\vec{K}_{\perp}$ is fixed. The role of such charge asymmetry and kinematic constraints in Pomeron-odderon DIS amplitudes has been noted previously for other final states $[115,116]$.

The two-gluon Pomeron and three-gluon odderon form factors were discussed previously in [117] albeit this work did not identify these form factors as such. More importantly, we have provided explicit first-principles expressions for the Pomeron form factor in Eq. (39) and likewise for the odderon form factor in Eq. (61) in terms of the QCD light front wave function for valence Fock states. Therefore exclusive measurements of the $J / \Psi$ at large $x_{\mathrm{Bj}}$ offer the opportunity to extract fundamental nonperturbative QCD physics contained in these wave functions.

It is important to note that by large $x_{\mathrm{Bj}}$, we have $x_{\mathrm{Bj}} \approx 0.1$ in mind. At larger values of $x_{\mathrm{Bj}}$, our approximations ignoring $\Delta x$ are no longer tenable. At smaller values of $x_{\mathrm{Bj}}<0.1$, higher gluon Fock states become important. While these can be incorporated in our approach, and matched eventually to the CGC EFT framework, their treatment is outside the scope of the present discussion.

We observe that while the exclusive $J / \Psi$ cross section is dominated by the Pomeron contribution, it can in principle be sensitive to the odderon form factor for particular kinematics. In contrast to the $J / \psi$ however, the $\eta_{c}$ meson with its $P=-1$ and $C=+1$ quantum numbers is dominantly produced in exclusive DIS by the three-gluon colorsinglet odderon exchange contribution. The exclusive $\eta_{c}$ production amplitude is simply

$$
\begin{aligned}
& \mathcal{A}_{T, L}^{\gamma^{*} p \rightarrow \eta_{c} p}\left(Q^{2}, \vec{K}_{\perp}\right) \\
& \quad \sim i \int \mathrm{d}^{2} r \int \frac{d z}{4 \pi}\left(\Psi_{\gamma^{*}} \Psi_{\eta_{c}}^{*}\right)\left(\vec{r}, z, Q^{2}\right) e^{-i \frac{(1-2 z)}{2} \vec{r} \cdot \vec{K}_{\perp}} i \mathcal{O}\left(\vec{r}, \vec{K}_{\perp}\right),
\end{aligned}
$$

where $\Psi_{\eta_{c}}$ is the light-cone $\eta_{c}$ wave function. Indeed, exclusive $\eta_{c}$ was proposed some time ago [118] as the cleanest channel for discovery of the odderon ${ }^{8}$ where the focus was on $\eta_{c}$ production at small $x_{\mathrm{Bj}}$ at HERA. The authors of Ref. [120] followed the approach of $[99,100]$ to estimate the HERA DIS $\eta_{c}$ cross section to be $47 \mathrm{pb}$ for photoproduction and $11 \mathrm{pb}$ for $Q^{2}=5 \mathrm{GeV}^{2}$. However the authors of $[99,100]$ expressed the odderon form factor in terms of that of the Pomeron form factor. Our study shows that this assumption is likely unjustified; we plan to investigate its quantitative impact in a future publication.

Searches at HERA did not reveal any evidence for exclusive $\eta_{c}$. From the theory perspective, this may be because the odderon amplitude is suppressed at small $x$. While not definitive, studies of the small $x$ evolution of the odderon suggest that its energy dependence is much

\footnotetext{
${ }^{8}$ For a nice review of both the theoretical work on the odderon and experimental searches, we refer the reader to [119].
}

smaller than that of the Pomeron [121]; it may even decrease with increasing energy $[122,123]$. Therefore, searches at larger values of $x_{\mathrm{Bj}}$ may be more promising. Further, since the cross section for such exclusive processes is small, such searches will benefit from the much higher luminosities at Jefferson Lab and in future at the EIC.

\section{SUMMARY AND OUTLOOK}

In this paper, we developed a novel formalism within the framework of light front QCD to compute color charge correlators and their associated color charge form factors. For simplicity, we constructed the quadratic $\langle\rho \rho\rangle$ and cubic $\langle\rho \rho \rho\rangle$ correlators of valence quark Fock states in the proton. The extension of our computation to include gluon and sea quark color charge densities is straightforward if more involved. These quadratic and cubic color charge correlators are precisely the color-singlet two-gluon Pomeron form factor and the three-gluon odderon form factor respectively. They capture important nonperturbative physics on the spatiotemporal distribution of color charges in the proton, and they offer a complementary description of this tomography to that offered by TMDs and GPDs. Further, they provide useful classical intuition at the level of the YangMills dynamics of QCD. As a striking example, note that the Wong equations [124] satisfied by classical color charges in background gauge fields are embedded in the structure of the QCD effective action [125]. Classical intuition at this level can motivate experimental searches for novel QCD effects.

While expressing observables in terms of expectation values of color charge correlators is uncommon at large $x$ (see however [126]), it is a key feature of the color glass condensate framework at small $x$, whereby dynamical manybody information from nonperturbative initial conditions is encoded in a gauge invariant density matrix $W[\rho]$. For a large nucleus, this quantity is the Gaussian weight functional of the McLerran-Venugopalan model. However, this formalism breaks down for the proton at large $x$ and the initial conditions for the small $x$ evolution of color charge correlations in the proton have a significant source of uncertainty.

We showed that exclusive measurements of quarkonia at large $x$ allow for independent extraction of $\langle\rho \rho\rangle$ and $\langle\rho \rho \rho\rangle$. Expectation values of these, and the associated Pomeron and odderon color charge form factors, can be extracted from clean exclusive DIS measurements of quarkonium final states at large $x$. These form factors, and in principle higher moments of the color charge density, therefore provide a bridge between small $x$ and large $x$ in QCD, one that is constrained by high energy proton-proton and protonnucleus experiments on multiparticle correlations at RHIC and LHC on the one hand, and DIS experiments at Jefferson Lab on the other. We also applied the formalism towards computing the gluon distribution of a proton and obtained sensible results. We anticipate that the Electron-Ion Collider, which will have an unparalleled combination of $x$ reach and high luminosities, will bring powerful new insight 
into the underlying dynamics of many-body color charge correlations in QCD.

Another interesting avenue of research that presents itself is the extraction of color charge correlations and form factors in polarized deep inelastic scattering and polarized protonproton collisions. Odderon exchange can for instance be probed in the single spin asymmetries measured in polarized proton-proton collisions [127]. Single spin asymmetries in semi-inclusive open charge production in polarized DIS are also sensitive to the odderon operator $[128,129]$. These connections between color charge form factors in a wide range of experiments are ripe for further exploration.

\section{ACKNOWLEDGMENTS}

This material is based on work supported by the U.S. Department of Energy, Office of Science, Office of Nuclear Physics, under Contracts No. DE-SC0012704 (A. D. and R. V.) and within the framework of the TMD Theory Topical Collaboration (R.V). A. D. also acknowledges support by the DOE Office of Nuclear Physics through Grant No. DEFG02-09ER41620 and from The City University of New York through the PSC-CUNY Research Grant No. 602620048. A. D. would also like to thank the Nuclear Theory Group of Brookhaven National Laboratory for kind hospitality. G. A. M. would like to thank the Lab for Nuclear Science at MIT, the Southgate Fellowship of Adelaide University (Australia), the Bathsheba de Rothchild Fellowship of Hebrew University (Jerusalem), the Shaoul Fellowship of Tel Aviv University, the Physics Division of Argonne National Laboratory and the U.S. Department of Energy Office of Science, Office of Nuclear Physics under Award No. DE-FG02-97ER-41014 for support that enabled this work. This work was also supported by the U.S. Department of Energy Office of Science, Office of Nuclear Physics under Awards No. DE-FG02-97ER-41014, No. DEFG02-94ER40818 and No. DE-FG02-96ER-40960; the Pazy Foundation; and by the Israel Science Foundation (Israel) under Grants No. 136/12 and No. 1334/16. We thank D. Kharzeev, L. Motyka and T. Stebel for useful comments on the manuscript. The figures in this paper have been prepared with Jaxodraw [130].

\section{APPENDIX A: $P^{+}$LIMIT OF THE DENSITY OPERATOR, AND THE PARTON PANCAKE}

Consider Eq. (23). This contains a term

$$
P^{+} e^{i\left(x_{q}-x_{p}\right) P^{+} r^{-}},
$$

which oscillates like crazy if $P^{+} \rightarrow \infty$ unless $r^{-}$and/or $\left(x_{p}-x_{q}\right)$ vanishes. In those cases the term is infinite. This is suggestive of delta functions and the pancake shape of high energy projectiles.

To better understand the term in Eq. (A1) consider a test function $f\left(r^{-}\right)$which is continuous at the origin and nonzero over a finite region of space. Such would arise in taking the matrix element of the density operator in the proton wave function. Then

$$
\begin{aligned}
& \int d r^{-} f\left(r^{-}\right) \lim _{P^{+} \rightarrow \infty} P^{+} e^{i\left(x_{q}-x_{p}\right) P^{+} r^{-}} \\
& =\lim _{P^{+} \rightarrow \infty} \int d u e^{i\left(x_{q}-x_{p}\right) u} f\left(u / P^{+}\right) \\
& =f(0) \int d u e^{i\left(x_{q}-x_{p}\right) u}=f(0) 2 \pi \delta\left(x_{q}-x_{p}\right) .
\end{aligned}
$$

Thus the term of Eq. (A1) and the density operator of Eq. (23) act as a delta function in both $x_{q}-x_{p}$ and $r^{-}$.

Thus effectively

$$
\lim _{P^{+} \rightarrow \infty} P^{+} e^{i\left(x_{q}-x_{p}\right) P^{+} r^{-}} \rightarrow 2 \pi \delta\left(x_{p}-x_{q}\right) \delta\left(r^{-}\right) .
$$

We therefore see that the density $\rho^{a}(r)$ contains $\delta\left(r^{-}\right)$, hence the pancake shape. Using Eq. (A3) in Eq. (23) and integrating over $r^{-}$leads immediately to Eq. (26).

The corrections of order $1 / P^{+}$can be understood from Eq. (A2), by using

$$
f\left(u / P^{+}\right) \approx f(0)+f^{\prime}(0) \frac{u}{P^{+}} .
$$

Including the second term gives a correction term:

$$
\frac{2 \pi i}{P^{+}} f^{\prime}(0) \frac{\partial}{\partial x_{q}} \delta\left(x_{q}-x_{p}\right)
$$

\section{APPENDIX B: THE ODDERON AMPLITUDE IN TERMS OF THE ODDERON FORM FACTOR}

The odderon contribution to the amplitude in Eq. (75) can be written out explicitly as

$$
\frac{1}{2 N_{c}} \operatorname{tr}\left\langle U\left(\vec{x}_{T}\right) U^{\dagger}\left(\vec{y}_{T}\right)-U\left(\vec{y}_{T}\right) U^{\dagger}\left(\vec{x}_{T}\right)\right\rangle_{K_{\perp}}=
$$




$$
\begin{gathered}
-\int d x^{-} \int_{x^{-}} d y^{-} \int d z^{-} A^{+}\left(x^{-}, \vec{x}_{T}\right) A^{+}\left(y^{-}, \vec{x}_{T}\right) A^{+}\left(z^{-}, \vec{y}_{T}\right) \\
+\int d x^{-} \int_{x^{-}} d y^{-} \int d z^{-} A^{+}\left(x^{-}, \vec{y}_{T}\right) A^{+}\left(y^{-}, \vec{y}_{T}\right) A^{+}\left(z^{-}, \vec{x}_{T}\right) \\
+\int d x^{-} \int_{x^{-}} d y^{-} \int_{y^{-}} d z^{-} A^{+}\left(x^{-}, \vec{x}_{T}\right) A^{+}\left(y^{-}, \vec{x}_{T}\right) A^{+}\left(z^{-}, \vec{x}_{T}\right) \\
-\int d x^{-} \int_{x^{-}} d y^{-} \int_{y^{-}} d z^{-} A^{+}\left(x^{-}, \vec{y}_{T}\right) A^{+}\left(y^{-}, \vec{y}_{T}\right) A^{+}\left(z^{-}, \vec{y}_{T}\right) \\
-\int d x^{-} \int^{x^{-}} d y^{-} \int^{y^{-}} d z^{-} A^{+}\left(x^{-}, \vec{y}_{T}\right) A^{+}\left(y^{-}, \vec{y}_{T}\right) A^{+}\left(z^{-}, \vec{y}_{T}\right) \\
+\int d x^{-} \int^{x^{-}} d y^{-} \int y^{y^{-}} d z^{-} A^{+}\left(x^{-}, \vec{x}_{T}\right) A^{+}\left(y^{-}, \vec{x}_{T}\right) A^{+}\left(z^{-}, \vec{x}_{T}\right)>
\end{gathered}
$$

With a little algebra one can combine Eqs. (B2) and (B5) to

$$
\begin{aligned}
\operatorname{tr} t^{a} t^{b} t^{c} & \int d x^{-} \int d y^{-} \int d z^{-} A^{+a}\left(z^{-}, x_{T}\right) A^{+b}\left(x^{-}, y_{T}\right) A^{+c}\left(y^{-}, y_{T}\right) \\
= & \frac{1}{4} d^{a b c} \int d x^{-} \int d y^{-} \int d z^{-} A^{+a}\left(z^{-}, x_{T}\right) A^{+b}\left(x^{-}, y_{T}\right) A^{+c}\left(y^{-}, y_{T}\right) .
\end{aligned}
$$

In the last step we have used that the factor multiplying $\operatorname{tr} t^{a} t^{b} t^{c}$ is symmetric under $b \leftrightarrow c$. Since all fields are now integrated over $x^{-}$without limits they can be traded for $\rho^{a}(q)$ from Eq. (25) so that the previous line becomes

$$
\frac{g^{3}}{4} d^{a b c} \int_{q_{1}} \int_{q_{2}} \int_{q_{3}} \frac{1}{q_{1}^{2}} \frac{1}{q_{2}^{2}} \frac{1}{q_{3}^{2}} e^{i\left(q_{1} \cdot x_{T}+\left(q_{2}+q_{3}\right) \cdot y_{T}\right)} \rho^{a}\left(q_{1}\right) \rho^{b}\left(q_{2}\right) \rho^{c}\left(q_{3}\right) .
$$

Along the same lines, the sum of (B3) and (B4) can be rewritten as

$$
\begin{aligned}
& -\frac{1}{4} d^{a b c} \int d x^{-} \int d y^{-} \int d z^{-} A^{+a}\left(z^{-}, y_{T}\right) A^{+b}\left(x^{-}, x_{T}\right) A^{+c}\left(y^{-}, x_{T}\right) \\
& =-\frac{g^{3}}{4} d^{a b c} \int_{q_{1}} \int_{q_{2}} \int_{q_{3}} \frac{1}{q_{1}^{2}} \frac{1}{q_{2}^{2}} \frac{1}{q_{3}^{2}} e^{i\left(q_{1} \cdot y_{T}+\left(q_{2}+q_{3}\right) \cdot x_{T}\right)} \rho^{a}\left(q_{1}\right) \rho^{b}\left(q_{2}\right) \rho^{c}\left(q_{3}\right) .
\end{aligned}
$$

The remaining terms from Eqs. (B6)-(B9) involve integrals over $A^{+}\left(x^{-}, x_{T}\right)$ at the same point $x_{T}$, i.e., integrals of the same (matrix valued) function $A^{+}\left(x^{-}\right)$. One may thus use standard identities for "time" ordered exponentials of a matrix $A(t)$ :

$$
T \int d t_{1} \cdots \int d t_{n} A\left(t_{1}\right) \cdots A\left(t_{n}\right)=T \int d t_{1} \cdots \int d t_{n} \frac{1}{n !} \sum_{\text {perm }} A\left(t_{i_{1}}\right) \cdots A\left(t_{i_{n}}\right) .
$$

The sum is over all permutations of $A\left(t_{1}\right), A\left(t_{2}\right), \ldots, A\left(t_{n}\right)$. We can now express (B6)+(B9) as

$$
\begin{aligned}
2 & \frac{1}{3 !} \frac{1}{4} d^{a b c} \int d x^{-} \int d y^{-} \int d z^{-} A^{+a}\left(x^{-}, \vec{x}_{T}\right) A^{+b}\left(y^{-}, \vec{x}_{T}\right) A^{+c}\left(z^{-}, \vec{x}_{T}\right) \\
& =\frac{g^{3}}{12} d^{a b c} \int_{q_{1}} \int_{q_{2}} \int_{q_{3}} \frac{1}{q_{1}^{2}} \frac{1}{q_{2}^{2}} \frac{1}{q_{3}^{2}} e^{i\left(q_{1}+q_{2}+q_{3}\right) \cdot x_{T}} \rho^{a}\left(q_{1}\right) \rho^{b}\left(q_{2}\right) \rho^{c}\left(q_{3}\right) .
\end{aligned}
$$

Similarly, the sum of (B7) and (B8) is given by 


$$
\begin{array}{r}
-\frac{1}{12} d^{a b c} \int d x^{-} \int d y^{-} \int d z^{-} A^{+a}\left(x^{-}, \vec{y}_{T}\right) A^{+b}\left(y^{-}, \vec{y}_{T}\right) A^{+c}\left(z^{-}, \vec{y}_{T}\right) \\
=-\frac{g^{3}}{12} d^{a b c} \int_{q_{1}} \int_{q_{2}} \int_{q_{3}} \frac{1}{q_{1}^{2}} \frac{1}{q_{2}^{2}} \frac{1}{q_{3}^{2}} e^{i\left(q_{1}+q_{2}+q_{3}\right) \cdot y_{T}} \rho^{a}\left(q_{1}\right) \rho^{b}\left(q_{2}\right) \rho^{c}\left(q_{3}\right) .
\end{array}
$$

[1] D. Boer et al., arXiv:1108.1713.

[2] J. Dudek et al., Eur. Phys. J. A 48, 187 (2012).

[3] X. d. Ji, Phys. Rev. Lett. 91, 062001 (2003).

[4] E. P. Wigner, Phys. Rev. 40, 749 (1932).

[5] D. Mueller, D. Robaschik, B. Geyer, F.-M. Dittes, and J. Horejsi, Fortschr. Phys. 42, 101 (1994).

[6] X. D. Ji, Phys. Rev. Lett. 78, 610 (1997).

[7] A. V. Radyushkin, Phys. Lett. B 380, 417 (1996).

[8] J. C. Collins, L. Frankfurt, and M. Strikman, Phys. Rev. D 56, 2982 (1997).

[9] M. Burkardt, Int. J. Mod. Phys. A 18, 173 (2003).

[10] M. Diehl, Phys. Rep. 388, 41 (2003).

[11] A. V. Belitsky and A. V. Radyushkin, Phys. Rep. 418, 1 (2005).

[12] J. P. Ralston and D. E. Soper, Nucl. Phys. B152, 109 (1979).

[13] J. C. Collins and D.E. Soper, Nucl. Phys. B193, 381 (1981); B213, 545(E) (1983).

[14] P. J. Mulders and R. D. Tangerman, Nucl. Phys. B461, 197 (1996); B484, 538(E) (1997).

[15] D. Boer and P. J. Mulders, Phys. Rev. D 57, 5780 (1998).

[16] A. V. Belitsky, X. Ji, and F. Yuan, Nucl. Phys. B656, 165 (2003).

[17] G. A. Miller, Phys. Rev. C 68, 022201 (2003); Nucl. Phys. News 18, 12 (2008); Phys. Rev. C 76, 065209 (2007).

[18] V. Punjabi, C. F. Perdrisat, M. K. Jones, E. J. Brash, and C. E. Carlson, Eur. Phys. J. A 51, 79 (2015).

[19] G. A. Miller, Phys. Rev. Lett. 99, 112001 (2007).

[20] G. A. Miller, Annu. Rev. Nucl. Part. Sci. 60, 1 (2010).

[21] C. E. Carlson and M. Vanderhaeghen, Phys. Rev. Lett. 100, 032004 (2008).

[22] M. Burkardt, Phys. Rev. D 62, 071503 (2000); 66, 119903 (E) (2002).

[23] F. Dominguez, C. Marquet, B. W. Xiao, and F. Yuan, Phys. Rev. D 83, 105005 (2011).

[24] E. Petreska, Int. J. Mod. Phys. E 27, 1830003 (2018).

[25] O. Hen, G. A. Miller, E. Piasetzky, and L. B. Weinstein, Rev. Mod. Phys. 89, 045002 (2017).

[26] G. A. Miller, M. D. Sievert, and R. Venugopalan, Phys. Rev. C 93, 045202 (2016).

[27] F. Winter, W. Detmold, A. S. Gambhir, K. Orginos, M. J. Savage, P. E. Shanahan, and M. L. Wagman, Phys. Rev. D 96, 094512 (2017).

[28] E. Iancu and R. Venugopalan, The color glass condensate and high-energy scattering in QCD, in Quark Gluon Plasma, edited by R.C. Hwa et al. (World Scientific, Singapore, 2004), Vol. 3, pp. 249-363.
[29] F. Gelis, E. Iancu, J. Jalilian-Marian, and R. Venugopalan, Annu. Rev. Nucl. Part. Sci. 60, 463 (2010).

[30] Yu. Kovchegov and E. Levin, Quantum Chromodynamics at High Energy (Cambridge University Press, Cambridge, England, 2012).

[31] J. P. Blaizot, Rep. Prog. Phys. 80, 032301 (2017).

[32] L. D. McLerran and R. Venugopalan, Phys. Rev. D 49, 2233 (1994).

[33] L. D. McLerran and R. Venugopalan, Phys. Rev. D 49, 3352 (1994).

[34] L. D. McLerran and R. Venugopalan, Phys. Rev. D 50, 2225 (1994).

[35] Y. V. Kovchegov, Phys. Rev. D 54, 5463 (1996).

[36] S. Jeon and R. Venugopalan, Phys. Rev. D 70, 105012 (2004).

[37] L. V. Gribov, E. M. Levin, and M. G. Ryskin, Phys. Rep. 100, 1 (1983).

[38] A. H. Mueller and J. w. Qiu, Nucl. Phys. B268, 427 (1986).

[39] A. Ayala, J. Jalilian-Marian, L. D. McLerran, and R. Venugopalan, Phys. Rev. D 52, 2935 (1995).

[40] J. Jalilian-Marian, A. Kovner, L. D. McLerran, and H. Weigert, Phys. Rev. D 55, 5414 (1997).

[41] A. H. Mueller, Nucl. Phys. B335, 115 (1990).

[42] A. H. Mueller, Nucl. Phys. B558, 285 (1999).

[43] L. D. McLerran and R. Venugopalan, Phys. Rev. D 59, 094002 (1999).

[44] Y. V. Kovchegov, Phys. Rev. D 60, 034008 (1999).

[45] R. Venugopalan, Acta Phys. Pol. B 30, 3731 (1999).

[46] J. Jalilian-Marian, A. Kovner, A. Leonidov, and H. Weigert, Phys. Rev. D 59, 014014 (1998).

[47] J. Jalilian-Marian, A. Kovner, and H. Weigert, Phys. Rev. D 59, 014015 (1998).

[48] E. Iancu, A. Leonidov, and L. D. McLerran, Nucl. Phys. A692, 583 (2001).

[49] E. Ferreiro, E. Iancu, A. Leonidov, and L. McLerran, Nucl. Phys. A703, 489 (2002).

[50] I. Balitsky, Nucl. Phys. B463, 99 (1996).

[51] H. Weigert, Nucl. Phys. A703, 823 (2002).

[52] J. P. Blaizot, E. Iancu, and H. Weigert, Nucl. Phys. A713, 441 (2003).

[53] K. Rummukainen and H. Weigert, Nucl. Phys. A739, 183 (2004).

[54] E. A. Kuraev, L. N. Lipatov, and V. S. Fadin, Sov. Phys. JETP 45, 199 (1977).

[55] I. I. Balitsky and L. N. Lipatov, Sov. J. Nucl. Phys. 28, 822 (1978).

[56] E. Iancu, K. Itakura, and L. McLerran, Nucl. Phys. A724, 181 (2003). 
[57] A. Dumitru, J. Jalilian-Marian, T. Lappi, B. Schenke, and R. Venugopalan, Phys. Lett. B 706, 219 (2011).

[58] T. Lappi and H. Mantysaari, Phys. Rev. D 88, 114020 (2013).

[59] J. Kuokkanen, K. Rummukainen, and H. Weigert, Nucl. Phys. A875, 29 (2012).

[60] J. L. Albacete, N. Armesto, J. G. Milhano, P. QuirogaArias, and C. A. Salgado, Eur. Phys. J. C 71, 1705 (2011).

[61] F. Gelis and R. Venugopalan, Nucl. Phys. A776, 135 (2006).

[62] F. Gelis and R. Venugopalan, Nucl. Phys. A779, 177 (2006).

[63] F. Gelis, T. Lappi, and R. Venugopalan, Int. J. Mod. Phys. E 16, 2595 (2007).

[64] F. Gelis, T. Lappi, and R. Venugopalan, Phys. Rev. D 78, 054019 (2008).

[65] F. Gelis, T. Lappi, and R. Venugopalan, Phys. Rev. D 78, 054020 (2008).

[66] Y. V. Kovchegov and A. H. Mueller, Nucl. Phys. B529, 451 (1998).

[67] A. Dumitru and L. D. McLerran, Nucl. Phys. A700, 492 (2002).

[68] K. Dusling, W. Li, and B. Schenke, Int. J. Mod. Phys. E 25, 1630002 (2016).

[69] B. Schenke and R. Venugopalan, Phys. Rev. Lett. 113, 102301 (2014).

[70] J. D. Bjorken, S. J. Brodsky, and A. Scharff Goldhaber, Phys. Lett. B 726, 344 (2013).

[71] K. Dusling, M. Mace, and R. Venugopalan, Proc. Sci., QCDEV2017 (2018) 039 [arXiv:1801.09704].

[72] L. McLerran and V. Skokov, Nucl. Phys. A959, 83 (2017).

[73] D. Boer, T. Van Daal, P. J. Mulders, and E. Petreska, J. High Energy Phys. 07 (2018) 140.

[74] Y. V. Kovchegov and V. V. Skokov, Phys. Rev. D 97, 094021 (2018).

[75] M. Mace, V. V. Skokov, P. Tribedy, and R. Venugopalan, Phys. Rev. Lett. 121, 052301 (2018).

[76] H. Mäntysaari and B. Schenke, Phys. Rev. Lett. 117, 052301 (2016).

[77] J. L. Albacete and A. Soto-Ontoso, Phys. Lett. B 770, 149 (2017).

[78] P. Bozek, W. Broniowski, and M. Rybczynski, Phys. Rev. C 94, 014902 (2016).

[79] S. J. Brodsky, H. C. Pauli, and S. S. Pinsky, Phys. Rep. 301, 299 (1998).

[80] L. Lukaszuk and B. Nicolescu, Lett. Nuovo Cimento 8, 405 (1973).

[81] G. Antchev et al. (TOTEM Collaboration), arXiv:1712 .06153 .

[82] S. J. Brodsky and G. P. Lepage, Adv. Ser. Dir. High Energy Phys. 5, 93 (1989).

[83] S. J. Brodsky, D. S. Hwang, B. Q. Ma, and I. Schmidt, Nucl. Phys. B593, 311 (2001).

[84] J. F. Gunion and D. E. Soper, Phys. Rev. D 15, 2617 (1977).

[85] F. Schlumpf, Phys. Rev. D 47, 4114 (1993); 49, 6246(E) (1994).

[86] M. R. Frank, B. K. Jennings, and G. A. Miller, Phys. Rev. C 54, 920 (1996).

[87] G. A. Miller, Phys. Rev. C 66, 032201 (2002).
[88] B. Pasquini and S. Boffi, Phys. Rev. D 76, 074011 (2007).

[89] B. Pasquini, S. Boffi, and P. Schweitzer, Mod. Phys. Lett. A 24, 2903 (2009).

[90] C. Lorce, B. Pasquini, and M. Vanderhaeghen, J. High Energy Phys. 05 (2011) 041.

[91] I. C. Cloet and G. A. Miller, Phys. Rev. C 86, 015208 (2012).

[92] A. Krasnitz, Y. Nara, and R. Venugopalan, Nucl. Phys. A717, 268 (2003).

[93] R. V. Gavai and R. Venugopalan, Phys. Rev. D 54, 5795 (1996).

[94] C. S. Lam and G. Mahlon, Phys. Rev. D 61, 014005 (1999).

[95] A. H. Mueller, arXiv:hep-ph/9911289.

[96] E. Iancu and L. D. McLerran, Phys. Lett. B 510, 145 (2001).

[97] A. H. Mueller, Phys. Lett. B 523, 243 (2001).

[98] Y. V. Kovchegov, L. Szymanowski, and S. Wallon, Phys. Lett. B 586, 267 (2004).

[99] M. Fukugita and J. Kwiecinski, Phys. Lett. 83B, 119 (1979).

[100] J. Czyzewski, J. Kwiecinski, L. Motyka, and M. Sadzikowski, Phys. Lett. B 398, 400 (1997); 411, 402(E) (1997).

[101] K. Hafidi, S. Joosten, Z. E. Meziani, and J. W. Qiu, FewBody Syst. 58, 141 (2017).

[102] S. Joosten and Z. E. Meziani, Proc. Sci., QCDEV2017 (2018) 017.

[103] S. Joosten, arXiv:1803.08615.

[104] A. Accardi et al., Eur. Phys. J. A 52, 268 (2016).

[105] V. P. Goncalves and W. K. Sauter, Phys. Rev. D 91, 094014 (2015).

[106] H. Kowalski, L. Motyka, and G. Watt, Phys. Rev. D 74, 074016 (2006).

[107] N. N. Nikolaev and B. G. Zakharov, Z. Phys. C 49, 607 (1991).

[108] Y. Hatta, B. W. Xiao, and F. Yuan, Phys. Rev. D 95, 114026 (2017).

[109] K. Roy and R. Venugopalan, J. High Energy Phys. 05 (2018) 013.

[110] J. Bartels, K. J. Golec-Biernat, and K. Peters, Acta Phys. Pol. B 34, 3051 (2003).

[111] Y. Hatta, E. Iancu, K. Itakura, and L. McLerran, Nucl. Phys. A760, 172 (2005).

[112] S. Jeon and R. Venugopalan, Phys. Rev. D 71, 125003 (2005).

[113] A. Dumitru, J. Jalilian-Marian, and E. Petreska, Phys. Rev. D 84, 014018 (2011); A. Dumitru and E. Petreska, Nucl. Phys. A879, 59 (2012).

[114] J. R. Forshaw and D. A. Ross, Quantum Chromodynamics and the Pomeron, Cambridge Lecture Notes in Physics (Cambridge University Press, Cambridge, England, 1997).

[115] S. J. Brodsky, J. Rathsman, and C. Merino, Phys. Lett. B 461, 114 (1999).

[116] P. Hägler, B. Pire, L. Szymanowski, and O. V. Teryaev, Eur. Phys. J. C 26, 261 (2002).

[117] S. J. Brodsky, E. Chudakov, P. Hoyer, and J. M. Laget, Phys. Lett. B 498, 23 (2001).

[118] A. Schäfer, L. Mankiewicz, and O. Nachtmann, in Proceedings of the Workshop Physics at HERA, Hamburg, 1991, edited by W. Buchmüller and G. Ingelman (unpublished).

[119] C. Ewerz, arXiv:hep-ph/0306137. 
[120] R. Engel, D. Y. Ivanov, R. Kirschner, and L. Szymanowski, Eur. Phys. J. C 4, 93 (1998).

[121] J. Bartels, L. N. Lipatov, and G. P. Vacca, Phys. Lett. B 477, 178 (2000).

[122] R. A. Janik and J. Wosiek, Phys. Rev. Lett. 82, 1092 (1999).

[123] T. Lappi, A. Ramnath, K. Rummukainen, and H. Weigert, Phys. Rev. D 94, 054014 (2016).

[124] S. K. Wong, Nuovo Cimento A 65, 689 (1970).
[125] J. Jalilian-Marian, S. Jeon, R. Venugopalan, and J. Wirstam, Phys. Rev. D 62, 045020 (2000).

[126] M. Burkardt, Phys. Rev. D 69, 057501 (2004).

[127] Y. V. Kovchegov and M. D. Sievert, Phys. Rev. D 86, 034028 (2012); 86, 079906(E) (2012).

[128] J. Zhou, Phys. Rev. D 89, 074050 (2014).

[129] H. Dong, D.-x. Zheng, and J. Zhou, arXiv:1805.09479.

[130] D. Binosi and L. Theuß1, Comput. Phys. Commun. 161, 76 (2004). 